\title{
Time lag in transient galactic and extragalactic accreting sources
}

\author{
Franco Giovannelli* \\ INAF - Istituto di Astrofisica e Planetologia Spaziali, Via del Fosso del Cavaliere, 100, 00133 \\ Roma, Italy \\ E-mail: Eranco.giovannellieiaps.inaf.it
}

\section{Gennady S. Bisnovatyi-Kogan}

IKI, Moscow, Russian Federation

E-mail: gkogandiki.rssi.ru

X-ray binaries are cauldrons of fundamental physical processes which appear along practically the whole electromagnetic spectrum. The sub-class of X-ray transient sources show multifrequency behaviour which deserve particular attention in order to understand the causing physics. These binary systems consist of a compact star and an optical star, therefore there is a mutual influence between these two stars that drive the low energy (LE) (i.e. radio, IR, optical) and high energy (HE) (i.e. UV, X-ray, $\gamma$-ray) processes. The LE processes are produced mostly on the optical star and the HE processes mostly on the compact star, typically a neutron star. Thus it appears evident that through the study of LE processes it is possible to understand also the $\mathrm{HE}$ processes and vice versa. In this paper we will discuss this problem starting from the experimental evidence of a delay between LE and HE processes detected for the first time in the X-ray/Be system A0535+26/HDE245770 (e.g. Giovannelli \& Sabau-Graziati, 2011; Giovannelli, Bisnovatyi-Kogan \& Klepnev, 2013 (here after GBK13); Giovannelli et al., 2015b). This delay is common in cataclysmic variables (CVs) and other binary systems with either a neutron star or a black hole.

Since a delay between LE processes and HE processes has been experimentally observed in several active galactic nuclei (AGNs), we will discuss also the tidal disruption of stars by massive BHs, following the original idea of Rees (1998): stars in galactic nuclei can be captured or tidally disrupted by a central black hole. Some debris would be ejected at high speed, the remainder would be swallowed by the hole, causing a bright flare lasting at most a few years.

The outline of this paper is:

- Antecedent fact

- Introduction

- X-ray Binary Systems

- Old \& News from the transient X-ray/Be system A0535+26/HDE245770

- The model for Galactic Accreting Sources

- The model for AGNs

- Discussion \& Conclusions

Accretion Processes in Cosmic Sources - II - APCS2018

3-8 September 2018

Saint Petersburg, Russian Federation 


\section{Antecedent Fact}

Figure 1 clearly explain all the mysteries of our Universe (Giovannelli, 2000). People who are able to read this sentence can understand that "The truth is written in the book of the Nature. We must learn to read this book".

$$
\begin{aligned}
& \text { 自然牥教科書には } \\
& \text { 真央が为子。 }
\end{aligned}
$$

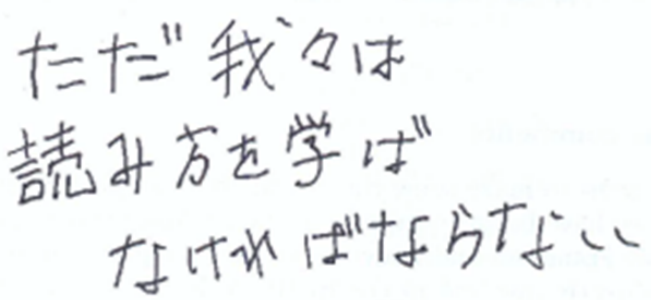

Figure 1: Understanding our Universe (adopted from Giovannelli, 2000).

The experiments provide the basic alphabet, immersed in an apparently chaotic soup, but necessary to understand the nature. From that soup we must extract words and phrases to compose the book of the nature. In other words, the data coming from the experiments constitute the basic alphabet that we use for constructing models that attempt to describe the nature. But we have a lot of models for interpreting the experimental data by the light of science. Depending on the hypotheses the results could run against the experiments. Then, in order to be acceptable, models can take into account and justify ALL the available data.

The same concept was expressed in much more incisive terms by Richard Phillips Feynman - Nobel laureate in Physics in 1965 - also known as The Great Explainer: It doesn't matter how beautiful your theory is, it doesn't matter how smart you are. If it doesn't agree with experiment, it's wrong.

The talk originating this paper had not been scheduled in the original Final Program of the workshop because the detailed description of the models that we will present is published in the proceedings of the Saint Petersburg workshop 2016 by G.S. Bisnovatyi-Kogan and F. Giovannelli, and other their publications. This talk came from the hole created in the program due to the sudden absence of Nazar Ikhsanov and Mateusz Wisniewicz. Therefore, instead of the details of the models, we will present the genesis of this work to the benefit above all of the young participants.

This paper is a demonstration of how we tried to read the book of the nature.

Undoubtedly the advent of new generation experiments ground- and space-based have given a strong impulse for verifying current theories, and for providing new experimental inputs for developing a new physics for going, probably, over the standard model (SM). Recent results coming

\footnotetext{
* Speaker.
} 
from Active Physics Experiments (APEs) - experiments in which we try to reproduce in a laboratory the physical conditions of processes occurring in nature we want to understand - and Passive Physics Experiments (PPEs) - experiments by which we observe the nature - have opened such a new path.

An extensive review on the situation about the knowledge of the physics of our Universe has been recently published by Giovannelli \& Sabau-Graziati (2016). The reader interested is invited to look at that paper.

The correct procedure in passive physics experiments is:

- to observe and collect experimental data;

- to analyze these data without any a priori bias;

- to attempt their interpretation on the base of current models;

- if not possible, it is mandatory to search for other models that cannot be "ad hoc".

This seems trivial, but, unfortunately, it is not so.

\section{Introduction}

Important observations started early in the past century with the discovery of inexplicable effects - the supernovae. They had been observed in ancient times but it was only with the establishing of the stellar and galactic distance scales that their true enormity was realized, namely the release of $\geq 10^{50} \mathrm{erg}$ within a matter of days.

The beginning of the Space Astrophysics Era is commonly located around the end of the fifties of the last century with the first space experiments, in the energy range $0.2-0.5 \mathrm{MeV}$, on board balloons. They were devoted to the detection of $\gamma$-rays generated in solar activity (Peterson $\&$ Winckler, 1958). But actually $\gamma$-ray astronomy was born in the last year of the XIXth century with the discoveries of penetrating gamma radiation (Villard, 1900), and the atmospheric ionization (Wilson, 1900). Wilson suggested that the extraterrestrial gamma radiation could be responsible for the atmospheric ionization. With balloon flights Hess (1912) demonstrated the extraterrestrial and extra-solar origin of the ionizing radiation, which was called Cosmic Rays.

Until 1927 it was thought that cosmic rays consisted of $\gamma$-rays. Thanks to the discovery of the dependence of the cosmic ray flux on the geomagnetic latitude during a trip from Java to Genoa made by Clay (1927), it was recognized that the composition of cosmic rays included charged particles. Later Hayakawa (1952) determined the contribution of $\gamma$-rays to the composition of cosmic rays as less than $1 \%$. The experiments outside the atmosphere started in 1946, soon after the end of the second world war, when the Naval Research Laboratory (NRL) launched a V2 rocket with a payload which observed the Sun's UV spectrum.

Since that time many space experiments were prepared and several fundamental results were reached. In our opinion the actual beginning of the Space Era for studying the Universe is the year 1962. An X-ray experiment - prepared by Giacconi, Gursky, Paolini \& Rossi - launched on board an Aerobee rocket discovered a strong X-ray emission from an extra-solar object, namely Sco X-1 (Giacconi et al., 1962). After this first historical experiment many others were launched on board 
rockets and later balloons and satellites. These experiments lead to our knowledge of an X-ray sky hitherto unknown which started to give experimental proofs of the first theories of Baade \& Zwicky (1934) about the possible existence of neutron stars.

Indeed, Baade \& Zwicky (1934) first suggested that the supernova was the result of the transition from a normal star to a neutron star. The essential point (Zwicky, 1939) being that the energy releases in such a process is comparable to the change in gravitational potential energy of a star, which collapses from its "normal" size of $\sim 10^{6} \mathrm{Km}$ down to the size of a neutron star of $\sim 10 \mathrm{Km}$.

In the 1950's, Burbidge et al. (1957) with their works on stellar nucleosynthesis suggested realistic models of stars prior to supernova explosion. The supernova process was seen as the result of catastrophic change of state occurring in the core of a highly evolved star, e.g. the transformation of an iron core into a helium core.

Contrary, Cameron (1958) suggested that this degenerate iron core would collapse to a neutron core through inverse beta decay.

The discovery (by chance) of the first X-ray source (Sco X-1) (Giacconi et al., 1962) ${ }^{1}$ accelerated the studies on neutron stars, until that Zel'dovich \& Guseinov (1965) suggested the presence of an unseen massive companion in a binary system.

Space orbiting observatories with larger and more sophisticated experiments - from Uhuru launched in 1970 (Giacconi et al., 1971) up to HEAO-1 launched in 1977 (Wood et al., 1984) discovered the most luminous galactic and extragalactic X-ray sources, such as pulsars, X-ray binaries, supernova remnants (SNRs), bursters, and active galactic nuclei (AGNs). But the qualitative jump in the observational capabilities was obtained with the HEAO-2 satellite (Einstein) (Giacconi et al., 1979) in which the X-ray focussing optics of the instruments enhanced the sensitivity in the soft X-ray range by a factor of about 1000 with respect to the old generation of detectors. Also the angular resolution was improved up to $\sim 2$ arcsec.

This allowed a re-definition of the positions of the already known X-ray sources and the discovery of a large number of weaker ones, such as the normal galaxies and normal stars spread on the entire HR diagram (Vaiana et al., 1981). The detected X-ray fluxes from these stars are definitively larger than those expected from theories of formation and heating of stellar coronae.

This led to a revolution in the comprehension of the role of the star rotation and of the magnetic field in the turbulent transport of energy from the nucleus to the external parts of a star.

Giovannelli (2016) published an extensive review about X-ray binary systems. Briefly we can summarize the main characteristics of these systems.

\section{X-ray binary systems}

The trivial definition of X-ray binaries (XRBs) is that they are binary systems emitting X-rays. However it has been largely demonstrated that X-ray binary systems emit energy in IR, Optical, UV, $\mathrm{X}$-ray, Gamma-ray and sometimes they show also valuable radio emission. They can be divided in different sub-classes

- High Mass X-ray Binaries (HMXB) in which the optical companion is an early type giant or supergiant star and the collapsed object is a neutron star or a black hole. They are concen-

\footnotetext{
${ }^{1}$ This was the first measurement that originated the Nobel Price of Riccardo Giacconi.
} 
trated around the galactic plane. The mass transfer is usually occurring via stellar wind; they show hard pulsed X-ray emission (from 0.069 to $1413 \mathrm{~s}$ ) with $\mathrm{KT} \geq 9 \mathrm{keV}$; typical X-ray luminosity is ranging from $10^{34}$ to $10^{39} \mathrm{erg} \mathrm{s}^{-1}$, and the ratio of X-ray to optical luminosity is $\sim 10^{-3}-10$. The HMXBs can be divided in two sub-classes

- Hard Transient X-ray Sources (HXTS) in which the neutron star is eccentrically (e $\sim 0.2-0.5$ ) orbiting around a V-III luminosity-class Be star ( $\mathrm{P}_{\text {orb }}>10$ days); they show strong variable pulsed hard $\mathrm{X}$-ray emission $\left(\mathrm{L}_{\mathrm{Xmax}} / \mathrm{L}_{\mathrm{X} \min }>100\right)$ with $\mathrm{KT} \geq 17 \mathrm{keV}$, and $\mathrm{P}_{\text {spin }}$ ranging from 0.069 to $1413 \mathrm{~s} ; \mathrm{L}_{\mathrm{X}}=10^{34}-10^{39} \mathrm{erg} \mathrm{s}^{-1}$.

- Permanent X-ray Sources in which the neutron star or black hole is circularly orbiting $(\mathrm{e} \sim 0)$ around a giant or supergiant $\mathrm{OB}$ star $\left(\mathrm{P}_{\text {orb }}<10\right.$ days); they show an almost steady permanent pulsed hard $\mathrm{X}$-ray emission $\left(\mathrm{L}_{\mathrm{Xmax}} / \mathrm{L}_{\mathrm{Xmin}} \ll 100\right)$, and $\mathrm{P}_{\text {spin }}$ ranging from 0.069 to $1413 \mathrm{~s} ; \mathrm{L}_{\mathrm{X}} \sim 10^{37} \mathrm{erg} \mathrm{s}^{-1}$.

- Supergiant X-ray Binaries (SGXBs): obscured sources, which display huge amount of low energy absorption produced by the dense wind of the supergiant companion, surrounded by a weakly magnetized neutron star.

- Supergiant Fast X-ray transients (SFXT), a subclass of SGXBs and a new subclass of transients in which the formation of transient accretion discs could be partly responsible for the flaring activity in systems with narrow orbits. They show $\mathrm{L}_{\text {Xpeak }} \approx 10^{36} \mathrm{erg} \mathrm{s}^{-1}$, and $\mathrm{L}_{\text {Xquiecence }} \approx 10^{32} \mathrm{erg} \mathrm{s}^{-1}$.

- Low Mass X-ray Binaries (LMXB) in which the optical companion is a low-mass-late-type star and the collapsed object is a neutron star or a black hole ( $\mathrm{P}_{\text {orb }}$ from 41 min to 11.2 days). They are concentrated in the globular clusters, and in the halo around the galactic center. The mass transfer in these systems is usually occurring via Roche lobe overflow. Their emission in soft X-ray range is usually not pulsed with $\mathrm{KT} \leq 9 \mathrm{keV}$. Their X-ray luminosity is ranging from $10^{36}$ to $10^{39} \mathrm{erg} \mathrm{s}^{-1}$ and $\mathrm{L}_{X} / \mathrm{L}_{\text {opt }} \sim 10^{2}-10^{4}$; many LMXBs show Quasi Periodic Oscillations (QPOs) between 0.02 and 1000 seconds and few of them also pulsed X-ray emission, such as Her X1, 4U 1626-27 and GX 1+4.

Many LMXB show transient behaviour in the form irregular X-ray bursts, when their luminosity increase several tens or hundreds times. During these luminous stages steady periodical signals, with milliseconds (ms) period, have been observed in several of them. In few of them ms X-ray pulsars have been discovered in quiescent stages between bursts. The ms $\mathrm{X}$-ray pulsars in LMXB form a link between binary X-ray sources and recycled binary radio pulsars, with ms periods and low magnetic fields (Bisnovatyi-Kogan and Komberg, 1974), which are formed on the place of these LMXB after ceasing of accretion, due to evolution of the companion star, transforming into low mass white dwarf, or a giant degenerate planet.

- Cataclysmic Variables (CVs) in which the optical companion is a low-mass-late-type star and the compact object is a white dwarf. The detected CVs are spread roughly around the solar system at distance of 200-300 pc. Orbital periods are ranging from tens of minutes to about ten hours. The mass transfer is occurring either via Roche lobe overflow or via accretion columns or in an intermediate way depending on the value of the magnetic field. 
Typical X-ray luminosity is ranging from $10^{32}$ to $10^{34} \mathrm{erg} \mathrm{s}^{-1}$. Updated reviews about CVs are those by Giovannelli (2008) and Giovannelli \& Sabau-Graziati (2015a);

- RS Canum Venaticorum (RS CVn) type systems, in which no compact objects are present and the two components are a $\mathrm{F}$ or $\mathrm{G}$ hotter star and a $\mathrm{K}$ star. Typical X-ray luminosity is ranging from $10^{30}$ to $10^{31} \mathrm{erg} \mathrm{s}^{-1}$. Usually in the current literature they are excluded from the class of X-ray binaries since historically they were discovered as X-ray emitters only with the second generation of X-ray experiments.

Figure 2 shows a compendium of the characteristics of the X-ray binaries (adapted from Giovannelli, 2015).

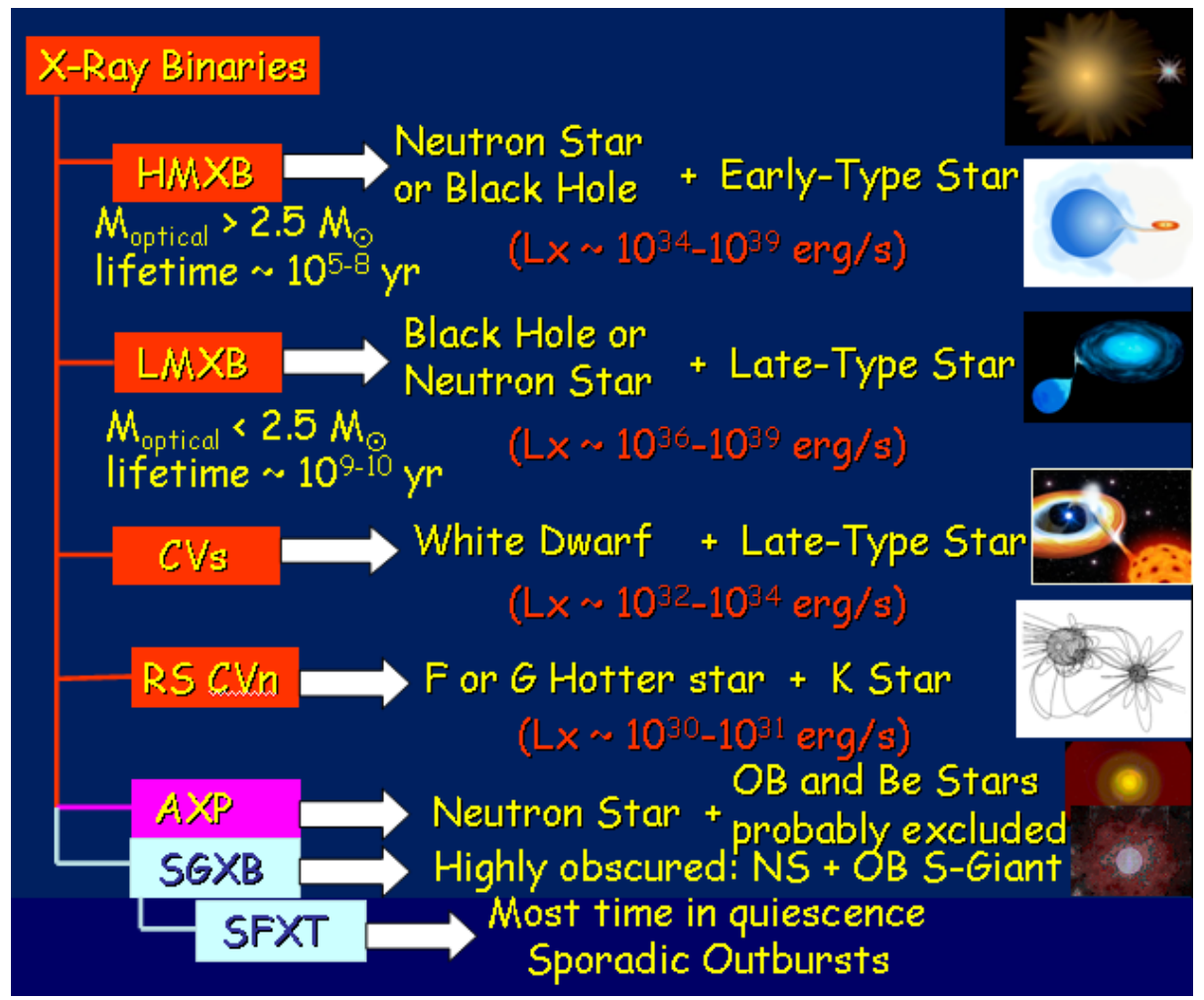

Figure 2: Classification of X-ray binaries (adapted from Giovannelli, 2015).

In binary systems there are essentially two ways for accreting matter from one star to the other: via accretion disk or via stellar wind (Giovannelli \& Sabau-Graziati, 2001, adapted from Blumenthal \& Tucker, 1974) (left panel of Fig 3). But in some cases there is a third way which is a mixture between the two, as for instance in eccentric binary systems close to the periastron passage where a temporary accretion disk can be formed around the neutron star (e.g. Giovannelli \& Ziółkowski (1990), like shown in the right panel of Fig. 3 (Giovannelli \& Sabau-Graziati, 2001, after Nagase, 1989).

XRBs are the best laboratory for the study of accreting processes thanks to their relative high luminosity in a large part of the electromagnetic spectrum. For this reason, multifrequency observations are fundamental in understanding their morphology and the physics governing their behaviour. 


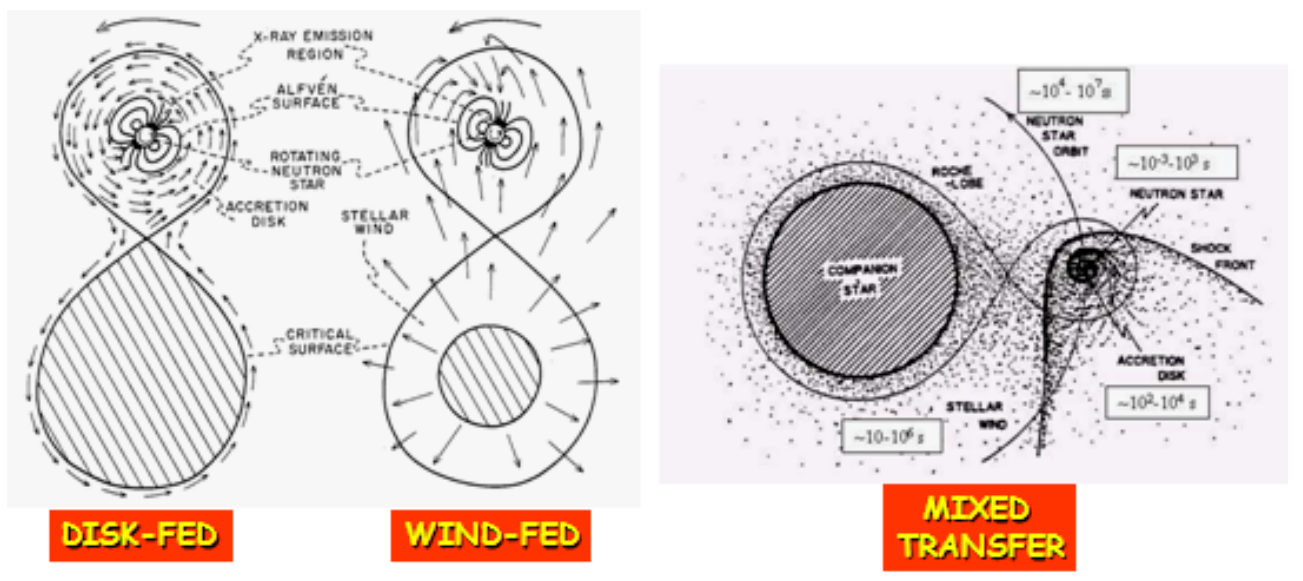

Figure 3: Left panel: accretion in X-ray binary systems disk-fed and wind-fed (adopted from Giovannelli \& Sabau-Graziati, 2001, adapted from Blumenthal \& Tucker, 1974). Right panel: mixed transfer (adopted from Giovannelli \& Sabau-Graziati, 2001, after Nagase, 1989).

Because of the strong interactions between the optical companion and collapsed object, low and high energy processes are strictly related.

Often, it is easier to perform observations of low energy processes (e.g. in radio, near-infrared (NIR) and optical bands) since the experiments are typically ground-based, on the contrary to observations of high energy processes, for which experiments are typically space-based.

\subsection{High Mass X-ray Binaries}

Among the X-ray binaries, the class of High Mass X-ray Binaries (HMXBs) constitutes an important group for studying the interactions either via stellar wind either via accretion disk between the optical and the compact companions.

Figure 4 shows schematically the classification of HMXBs (adopted from Giovannelli, 2015).

\subsection{X-ray/Be systems}

The X-ray/Be binaries are the most abundant group of massive X-ray binaries in the galaxy, with a total inferred number of between $10^{3}$ and $10^{4}$. The ones which do occasionally flare-up as transient X-ray/Be systems are only the "tip" of this vast "iceberg" of systems (van den Heuvel and Rappaport, 1987). The mass loss processes are due to the rapid rotation of the Be star, the stellar wind and, sporadically, to the expulsion of casual quantity of matter essentially triggered by gravitational effects close to the periastron passage of the neutron star. The long orbital period $(>10$ days) and a large eccentricity of the orbit $(>0.2)$ together with transient hard X-ray behavior are the main characteristics of these systems. Among the whole sample of galactic systems containing 114 X-ray pulsars (Liu, van Paradijs \& van den Heuvel, 2006), only few of them have been extensively studied. Among these, the system A 0535+26/HDE 245770 - HDE 245770 was nicknamed Flavia star by Giovannelli \& Sabau-Graziati, 1992) - is the best known thanks to concomitant favorable causes, which rendered possible forty three years of coordinated multifrequency observations, most of them discussed in the past by e.g. Giovannelli \& Sabau-Graziati (1992), Burger et al. (1996), 


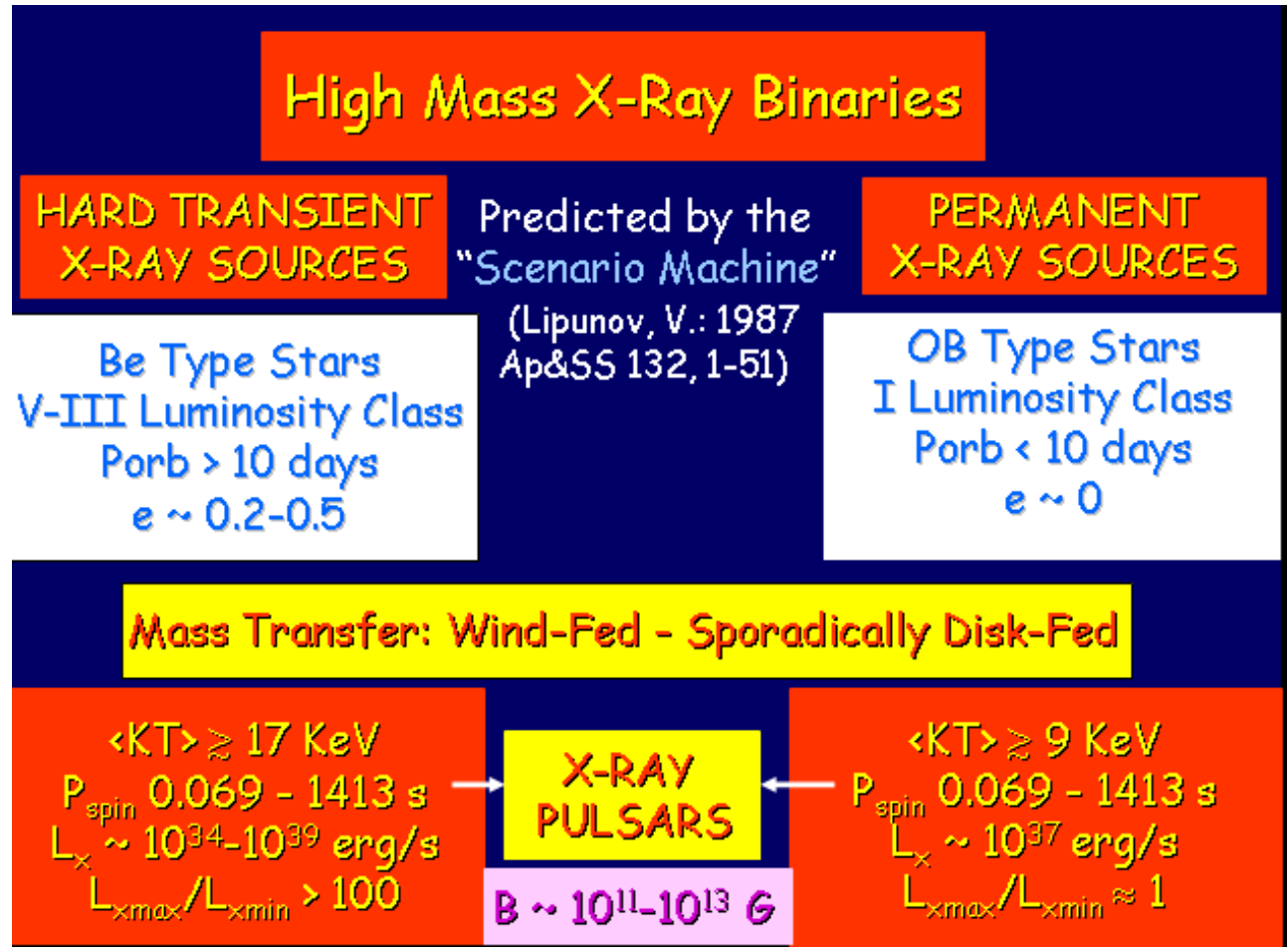

Figure 4: Classification of HMXBs (adopted from Giovannelli, 2015).

Piccioni et al. (1999), and later by Giovannelli \& Sabau-Graziati (2011) and Giovannelli et al. (2015a,b). Accretion powered X-ray pulsars usually capture material from the optical companion via stellar wind, since this primary star generally does not fill its Roche lobe. However, in some specific conditions (e.g. the passage at the periastron of the neutron star) and in particular systems (e.g. A $0535+26 / \mathrm{HDE} 245770$ ), it is possible the formation of a temporary accretion disk around the neutron star behind the shock front of the stellar wind. This enhances the efficiency of the process of mass transfer from the primary star onto the secondary collapsed star, as discussed by Giovannelli \& Ziolkowski (1990) and by Giovannelli et al. (2007) in the case of A 0535+26.

Optical emission of HMXBs is dominated by that of the optical primary component, which is not, in general, strongly influenced by the presence of the X-ray source. The behavior of the primary stars can be understood in the classical (or almost) frame-work of the astrophysics of these objects, i.e. by the study of their spectra which will provide indications on mass, radius, and luminosity. Both groups of HMXBs (transient and permanent) differ because of the different origin of the mass loss process: in the first, the mass loss process occurs via a strong stellar wind and/or because of an incipient Roche lobe over-flow; in the second group, the mass transfer is probably partially due to the rapid rotation of the primary star and partially to stellar wind and sporadically to expulsions of a casual quantity of matter, essentially triggered by gravitational effects because of periastron passage where the effect of the secondary collapsed star is more marked. A relationship between orbital period of HMXBs and the spin period of the X-ray pulsars is shown in Fig. 5 (updated from Giovannelli \& Sabau-Graziati, 2001 and from Corbet, 1984, 1986). It allows to 
recognize three kinds of systems, namely disk-fed, wind-fed $\left[\mathrm{P}_{\text {pulse }} \propto\left(\mathrm{P}_{\mathrm{orb}}\right)^{4 / 7}\right]$, and $\mathrm{X}$-ray/Be systems $\left[\mathrm{P}_{\text {pulse }} \propto\left(\mathrm{P}_{\text {orb }}\right)^{2}\right]$.

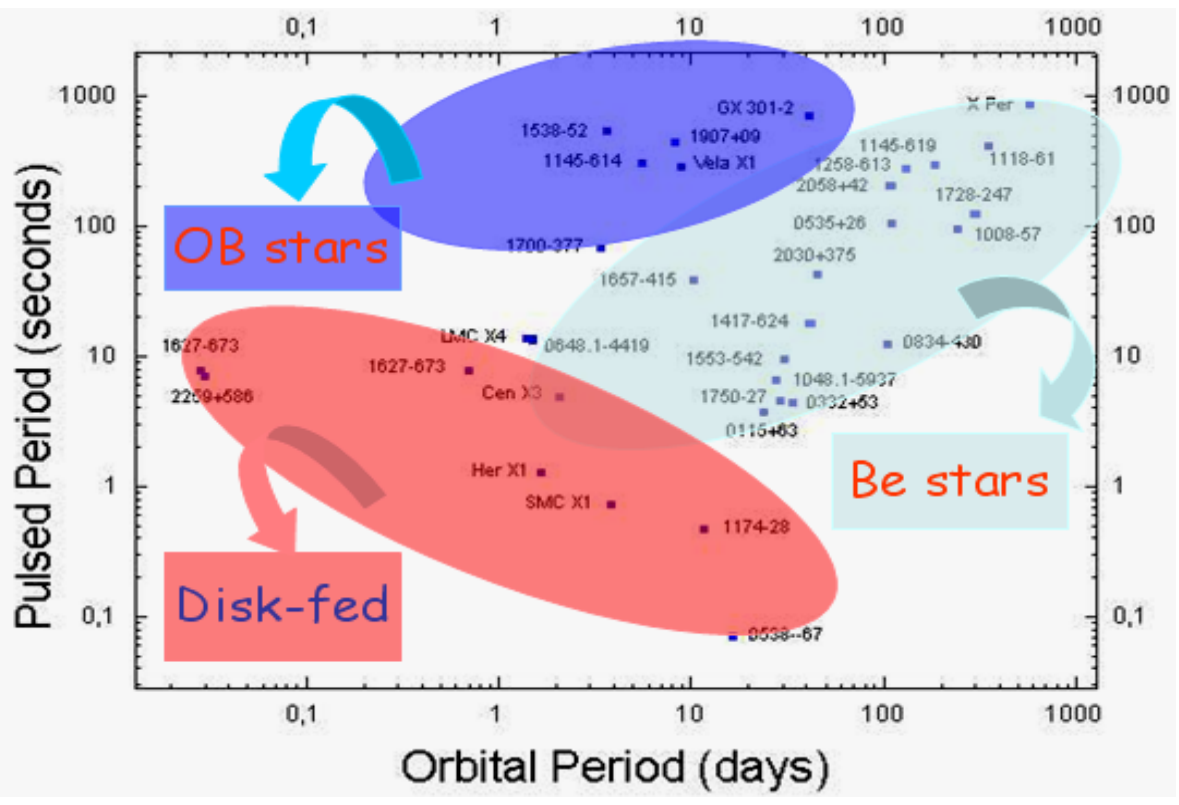

Figure 5: Spin period vs orbital period for X-ray pulsars. Disk-fed systems are clearly separated by systems having as optical counterparts either OB stars or Be stars (adopted from Giovannelli \& Sabau-Graziati, 2001, after Corbet, 1984, 1986).

Most of the systems having a Be primary star are hard X-ray $(\mathrm{KT}>10 \mathrm{KeV})$ transient sources (HXTS). They are concentrated on the galactic plane within a band of $\sim 3.9^{\circ}$. The orbits are quite elliptic and the orbital periods large (i.e. A 0538-66: e $=0.7, \mathrm{P}_{\text {orb }}=16.6$ days (Skinner et al., 1982); A 0535+26: e $=0.47$ (Finger, Wilson \& Hagedon, 1994), $P_{\text {orb }}=111.0$ days (Priedhorsky \& Terrell, 1983). The X-ray flux during outburst phases is of order 10-1000 times greater than during quiescent phases. For this reason, on the contrary, the stars belonging to the class of permanent $\mathrm{X}$-ray sources, which do not present such strong variations in X-ray emission, can be also named "standard" high mass X-ray binaries. In X-ray/Be systems, the primary Be star is relatively not evolved and is contained within its Roche lobe. The strong outbursts occur almost periodically in time scales of the order of weeks-months. Their duration is shorter than the quiescent phases. During X-ray outbursts, spin-up phenomena in several systems have been observed (i.e. A $0535+26$ and 4U 1118-61 (Rappaport \& Joss, 1981). The observed spin-up rates during the outbursts are consistent with torsional accretion due to an accretion disk (e.g. Ghosh, 1994). So, the formation of a temporary accretion disk around the collapsed object should be possible during outburst phases (e.g. Giovannelli \& Ziolkowski, 1990).

\section{Old \& News from the transient X-ray/Be system A0535+26/HDE245770}

The most studied HMXB system, for historical reasons and due to concomitant favourable causes, is the X-ray/Be system A $0535+26 / \mathrm{HDE} 245770$. By means of long series of coordinated multifrequency measurements, very often simultaneously obtained, it was possible to: 
- identify the optical counterpart HDE 245770 of the X-ray pulsar;

- identify various X-ray outbursts triggered by different states of the optical companion and influenced by the orbital parameters of the system;

- identify the presence of a temporary accretion disc around the neutron star at periastron.

Multifrequency observations of A $0535+26$ started soon after its discovery as an X-ray pulsar by the Ariel-5 satellite on April 14, 1975 (Coe et al., 1975). The X-ray source was in outburst with intensity of $\sim 2$ Crab and showed a pulsation of $\sim 104$ s (Rosenberg et al., 1975). The hard X-ray spectrum during the decay from the April 1975 outburst became softer, so that the 19 May spectrum had $E^{-0.8}$ and the 1 June spectrum $E^{-1.1}$ (Ricketts et al., 1975). Between 13 and 19 April, 1975 , as the nova brightened, the spectra showed some evidence of steepening. The best fit of the experimental data between roughly 27 and 28 April was compatible with an $8 \mathrm{keV}$ black-body curve (Coe et al., 1975). The X-ray source decayed from the outburst with an $e$-folding time of 19 days in the energy range of 3-6 keV (Kaluzienski et al., 1975).

In the X-ray error box of the X-ray source A 0535+26, detected by Ariel V, were present 11 stars up to $23^{\text {rd }}$ magnitude and one of them (HDE 245770) of magnitude around 9 showed the $\mathrm{H}_{\alpha}$ and $\mathrm{H}_{\beta}$ in emission, $\mathrm{H}_{\gamma}$ filled in with emisssion, and $\mathrm{H}_{\delta}, \mathrm{H}_{\mathcal{E}}, \ldots, \mathrm{H}_{10}$ in absorption (Margon et al., 1977). A priori probability of finding a 9 mag star in such a field is 0.004 , thus HDE 245770 was considered as the probable optical counterpart of A0535+26.

But in order to really associate this star with the X-ray pulsar, it was necessary to find a clear signature proving that the two objects would belong to the same binary system. This happened thanks to a sudden insight of one of us (FG), who predicted the fourth X-ray outburst of A $0535+26$ around mid December 1977. For this reason, Giovannelli's group was observing in optical HDE 245770 around the predicted period for the X-ray outburst of A $0535+26$. Figure 6 shows the Xray flux intensity of A $0535+26$ as deduced by various measurements available at that time, with obvious meaning of the symbols used (Giovannelli, 2005). FG's intuition was sparked by looking at the rise of the X-ray flux (red line) and at the 24th May 1977 measurement (red asterisk): he assumed that the evident rise of the X-ray flux would have produced an outburst similar to the first one, which occurred in 1975. Then with a simple extrapolation he predicted the fourth outburst, similar to the second: and this happened!

Optical photoelectric photometry of HDE 245770 showed significant light enhancement of the star relative to the comparison star BD +26 876 between Dec. 17 and Dec 21 (here after 771220-E) and successive fading up to Jan. 6 (Bartolini et al., 1978), whilst satellite SAS-3 was detecting an X-ray flare (Chartres \& Li, 1977). The observed enhancement of optical emission followed by the flare-up of the X-ray source gave a direct argument strongly supporting the identification of HDE 245770 - later nicknamed Flavia' star by Giovannelli \& Sabau-Graziati (1992) - with A 0535+26.

Soon after, with spectra taken at the Loiano $152 \mathrm{~cm}$ telescope with a Boller \& Chivens 26767 grating spectrograph (831 grooves/mm II-order grating: $39 \AA \mathrm{mm}^{-1}$ ) onto Kodak 103 aO plates, it was possible to classify HDE 245770 as O9.7IIIe star. This classification was so good that it survives even to the recent dispute attempts made with modern technology. The mass and radius of the star are $15 \mathrm{M}_{\odot}$ and $14 \mathrm{R}_{\odot}$, respectively; the distance to the system is $1.8 \pm 0.6 \mathrm{kpc}$ (Giangrande et al., 1980). 


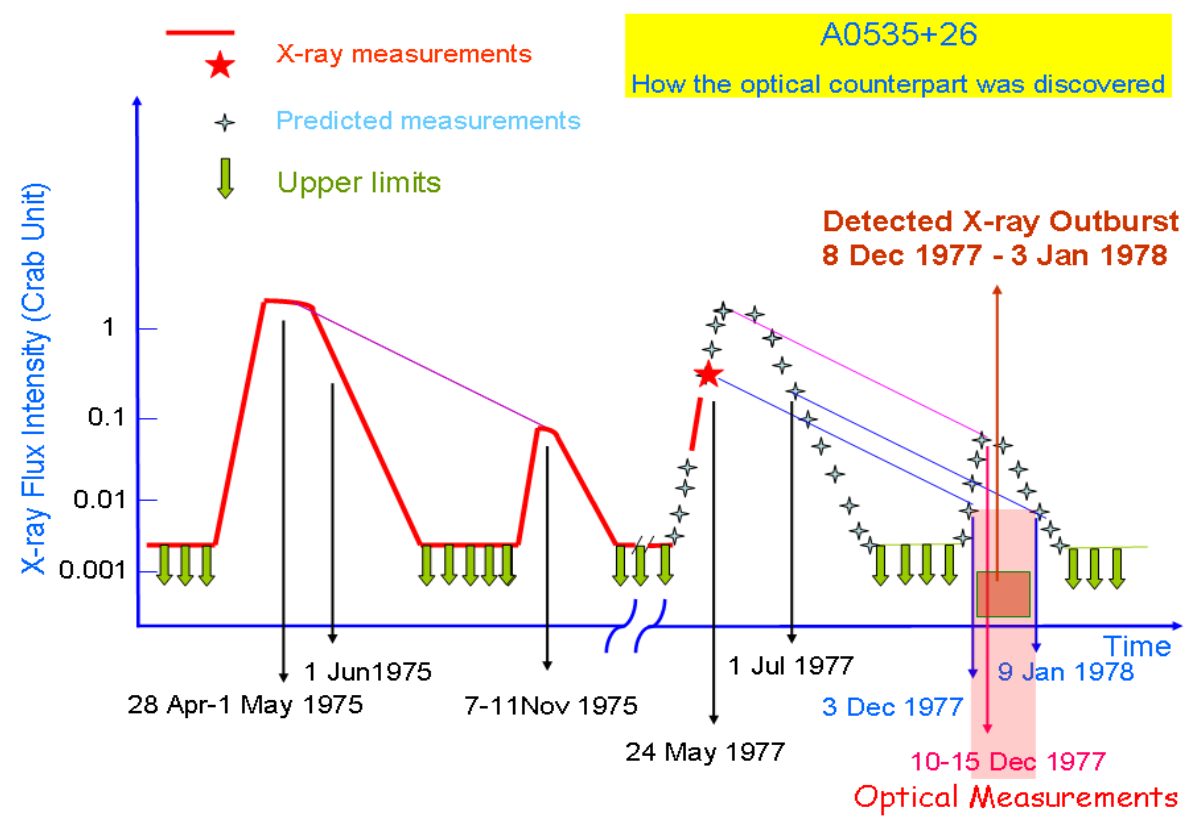

Figure 6: X-ray flux versus time of A $0535+26$. X-ray measurements are reported with red lines and asterisk, upper limits with green arrows, and predicted fluxes with light blue stars. Periods of real detected X-ray outburst and optical measurements are also marked (adopted from Giovannelli, 2005).

UV spectra taken with the IUE enabled the reddening of the system to be determined as $\mathrm{E}$ (B$\mathrm{V})=0.75 \pm 0.05 \mathrm{mag}$, the rotational velocity of the O9.7IIIe $\operatorname{star}\left(\mathrm{v}_{\text {rot }} \sin i=230 \pm 45 \mathrm{~km} \mathrm{~s}^{-1}\right)$, the terminal velocity of the stellar wind $\left(\mathrm{v}_{\infty} \simeq 630 \mathrm{~km} \mathrm{~s}^{-1}\right)$, the mass loss rate $\left(\dot{\mathrm{M}} \sim 10^{-8} \mathrm{M}_{\odot} \mathrm{yr}^{-1}\right.$ in quiescence (Giovannelli et al., 1982). During the October 1980 strong outburst, the mass loss rate was $\dot{\mathrm{M}} \sim 7.7 \times 10^{-7} \mathrm{M}_{\odot} \mathrm{yr}^{-1}$ (de Martino et al., 1989).

Complete reviews of this system can be found in Giovannelli et al. (1985), Giovannelli \& Sabau-Graziati (1992), and Burger et al. (1996).

Briefly, the properties of this system, placed at a distance of $1.8 \pm 0.6 \mathrm{kpc}$ (Giangrande et al., 1980), can be summarized as follows: a hard X-ray transient, long-period X-ray pulsar - the secondary star - is orbiting around the primary O9.7IIIe star. The masses are of $\sim 1.5 \pm 0.3 \mathrm{M}_{\odot}$ (Joss \& Rappaport 1984; Thorsett et al. 1993; van Kerkwijk, van Paradijs, J. \& Zuiderwijk, 1995), and $15 \mathrm{M}_{\odot}$ (Giangrande et al., 1980) for the secondary and primary star, respectively. The eccentricity is e $=0.47$ (Finger et al., 1994). Usually the primary star does not fill its Roche lobe (de Loore et al., 1984). However, the suggestion that there might be a temporary accretion disk around the X-ray pulsar when it approaches periastron (Giovannelli \& Ziółkowski, 1990) was confirmed by the X-ray measurements of Finger, Wilson \& Harmon (1996) and was discussed by Giovannelli et al. (2007).

The first suggestion of Bartolini et al. (1983) about the value of the orbital period $\left(\mathrm{P}_{\text {orb }}=\right.$ $110.856 \pm 0.002$ days), allowed Giovannelli \& Sabau-Graziati (2011) to discover a systematic delay ( $\sim 8$ days) of the X-ray outbursts with respect to the periastrons passages of the neutron star. Just a little before or simultaneously to the periastron, the system experiences an optical brightening 
ranging from $\approx 0.02$ to $\approx 0.2$ magnitudes.

The trigger of this discovery was the optical flare occurred at JD 2,444,944 (5th December 1981) (hereafter 811205-E; E stands for event) (Giovannelli et al., 1985) and followed by a short X-ray outburst (811213-E) (Nagase et al., 1982) - predicted by a private communication of Adriano Guarnieri (member of Giovannelli's group) to the team of Hakucho Japanise X-ray satellite. Unfortunately in the following years simultaneous optical and X-ray measurements were not always obtained around periastron passage. However, the available data were sufficient for showing the aforementioned systematic delay.

In order to explain and describe the 8-day delay between periastron passage and X-ray outbursts, GBK13 constructed a model adopting the orbital period determined by Priedhorsky \& Terrell (1983) from X-ray data $\left(\mathrm{P}_{\text {orb }}=111.0 \pm 0.4\right.$ days $)$, and the ephemeris $\mathrm{JD}_{\text {opt-outb }}=\mathrm{JD}_{0}(2,444,944)$ $\pm \mathrm{n}(111.0 \pm 0.4)$ days; the 111-day orbital period agrees within the error bars with many other determinations reported in the literature (from optical data, e.g. Guarnieri et al., 1985; de Martino et al., 1985; Hutchings, 1984; Janot-Pacheco, Motch \& Mouchet, 1987). From X-ray data (e.g. Nagase et al., 1982; Priedhorsky \& Terrell, 1983; Motch et al., 1991; Finger, Wilson \& Harmon, 1996; Coe et al., 2006; Finger et al., 2006).

In the following we will discuss the experimental results that corroborate GBK13 model, by the analysis of several results coming from multifrequency LE and HE observations of A 0535+26/HDE 245770 nicknamed Flavia' star.

\section{Time delay between optical and X-ray outbursts in A $0535+26 / H D E 245770$}

A description of the time-delay among many optical and X-ray events occurring around the periastron passages in the system A $0535+26 / \mathrm{HDE} 245770$ have been presented in the papers by GBK13 and Giovannelli et al. (2015a,b). However, just to remark the importance of the experimental evidence of such a time-delay we will present a few more examples that in our opinion definitively support the validity of the model developed in GBK13. Briefly, the model is the following: in the vicinity of periastron the mass flux $\dot{M}$ increases (depending on the activity of the Be star) between $\approx 10^{-8}$ and $\approx 10^{-7} \mathrm{M}_{\odot} \mathrm{yr}^{-1}$. The outer part of the accretion disk - geometrically thin and optically thick without advection (Shakura \& Sunyaev, 1973; Bisnovatyi-Kogan, 2011) becomes hotter, therefore the optical luminosity $\left(\mathrm{L}_{\mathrm{opt}}\right)$ increases. Due to large turbulent viscosity, the wave of the large mass flux is propagating toward the neutron star, thus the X-ray luminosity $\left(\mathrm{L}_{\mathrm{X}}\right)$ increases due to the appearance of a hot accretion disk region and due the accretion flow channeled by the magnetic field lines onto magnetic poles of the neutron star. The time-delay $\tau$ is the time between the optical and X-ray flashes appearance. Figure 7 shows a sketch of this model.

By using the ephemerides given by GBK13, namely:

$$
\mathrm{JD}_{\text {opt-outb }}=\mathrm{JD}_{0}(2,444,944) \pm \mathrm{n}(111.0 \pm 0.4) \text { days }
$$

that fixed the reference point at the date 5th December 1981 (811205-E), it was possible to explain the behaviour of the system during the year 2014 (Giovannelli et al., 2015a). It was possible not only to predict the arrival time of the X-ray outbursts following the optical flashes, but also the intensity $I_{X}$ of the X-ray flares, thanks to the relationship $I_{X}$ versus $\Delta V_{\text {mag }}$, where $\Delta V_{\text {mag }}$ is the relative variations of the $\mathrm{V}$ magnitude of the $\mathrm{Be}$ star around the periastron passage with respect 


\section{Accretion Disk geometrically thin and optically thick without advection (Shakura \& Sunyaev, 1973; Bisnovatyi-Kogan, 2002)}

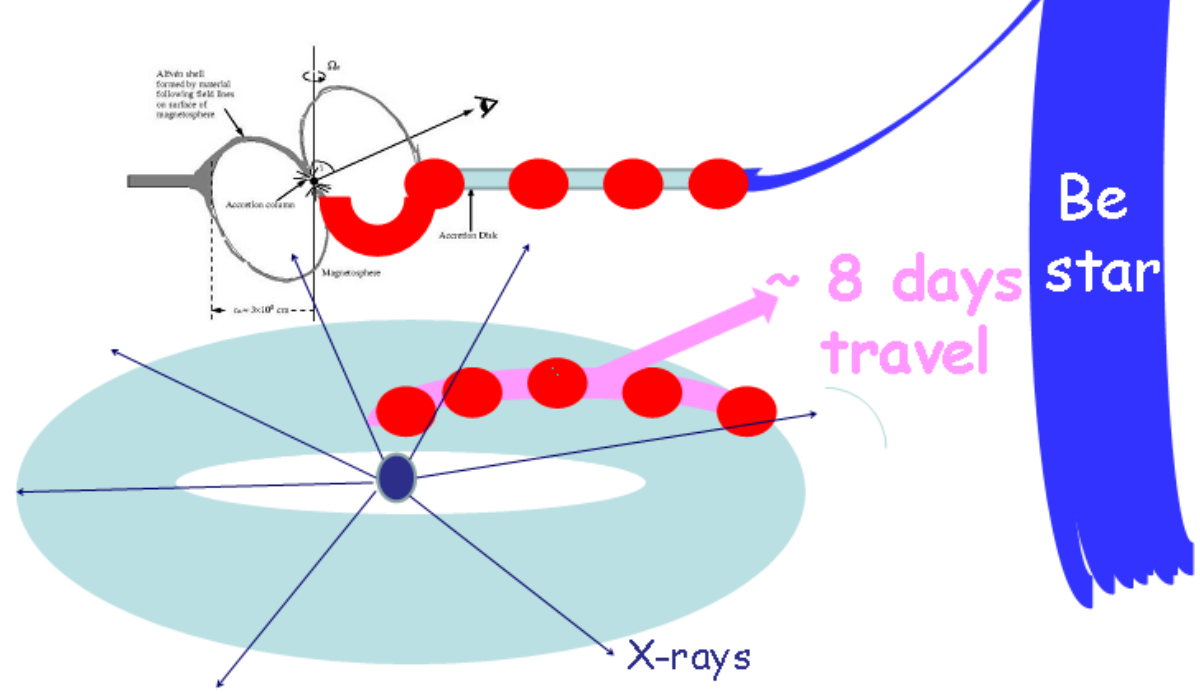

Figure 7: Sketch of the viscous accretion disk model for explaining the time-delay between X-ray and optical flashes (adopted from GBK13).

to the level before and after such a passage. This relationship is shown in Fig. 8 (adapted from Giovannelli et al., 2015b).

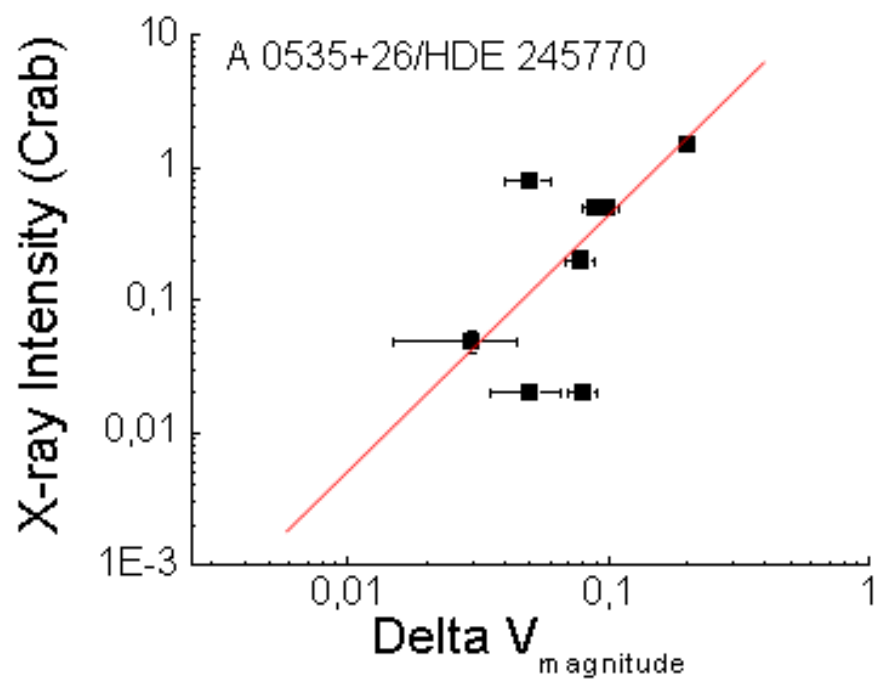

Figure 8: Intensity of the X-ray flare of A $0535+26$ versus the variation of V magnitude of HDE 245770 around the periastron passage (adapted from Giovannelli et al., 2015b).

We have also found a relationship between the equivalent width (EW) of $\mathrm{H}_{\alpha}$ and $\mathrm{I}_{\mathrm{x}}$. The values of $\mathrm{H}_{\alpha}$-EW have been taken only if measurements were performed around the periastron passage \pm 10 days. Unfortunately few measurements have been found in this time range. However, 
the trend of the relationship is rather good, as shown in Fig. 9, where data taken from CameroArranz et al. (2012), Yan, Li \& Liu (2012), Giovannelli et al. (2015b) are reported (Fasano, 2015). It is interesting to note that in one occasion, at the 106th periastron passage (JD 2,456,710 $=21$ Feb 2014) after 811205-E, optical photometry and spectroscopy as well as X-ray measurements from different experiments were obtained. A jump in the $\mathrm{H}_{\alpha}$-EW and $\mathrm{H}_{\beta}$-EW in correspondence with the rise of X-ray intensity was detected, being the jump of $\mathrm{H}_{\beta}$-EW delayed of $\approx 5$ days with respect to that of $\mathrm{H}_{\alpha}$-EW. This important result deserves further investigations. However, the jumps of $\mathrm{H}_{\alpha}-\mathrm{EW}$ and $\mathrm{H}_{\beta}$-EW could originate because of a contribution to the total emission in those lines coming from the temporary accretion disk around the neutron star (Giovannelli et al., 2015a, and the references therein). And if so, the delay between $\mathrm{H}_{\beta}-\mathrm{EW}$ and $\mathrm{H}_{\alpha}$-EW jumps should be explicable within the framework of GBK13's model.

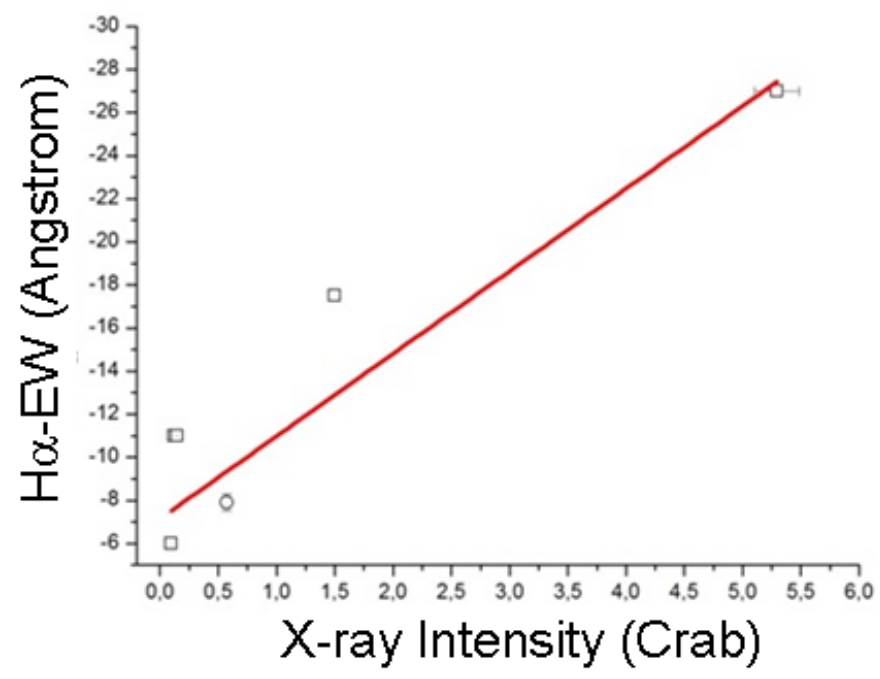

Figure 9: Intensity of the X-ray flare of A $0535+26$ versus the equivalent width of $\mathrm{H}_{\alpha}$ of HDE 245770 around the periastron passage (data taken from Camero-Arranz et al., 2012; Yan, Li \& Liu, 2012; Giovannelli et al., 2015b) (figure adopted from Fasano, 2015).

An impressive strong optical event have been detected on March 19, 2010 (JD 2,455,275). On the basis of such a strong optical activity - especially $\mathrm{H}_{\gamma}$ in emission - Giovannelli, Gualandi \& Sabau-Graziati (2010, ATel 2497) predicted the incoming X-ray outburst of A 0535+26, which actually occurred (Caballero et al., 2010b, ATel 2541). The X-ray intensity reached was $1.18 \mathrm{Crab}$ on April 3, 2010 in the range 15-50 keV of BAT/SWIFT (Caballero et al., 2010a,b,c,d; Caballero et al., 2011). Figure 10 shows the March-April 2010 event. The X-ray flare started about 8 days after the 93th periastron passage after the $811205-\mathrm{E}$, just when optical spectroscopy was performed by Giovannelli, Gualandi \& Sabau-Graziati (2010), and reached the maximum about 12 days later and decayed in about 20 days roughly as occurred in 1975 when A0535+26 was discovered by the Ariel V satellite.

The astonishing fact that definitively demonstrate the goodness of GBK13's ephemerides and the mechanism triggering the X-ray outburst with a time-delay with respect to the optical flare around the periastron passage is reported in Fig. 11.

Indeed, in Fig. 11 the measurements of the first detection of A $0535+26$ by Ariel V satellite 


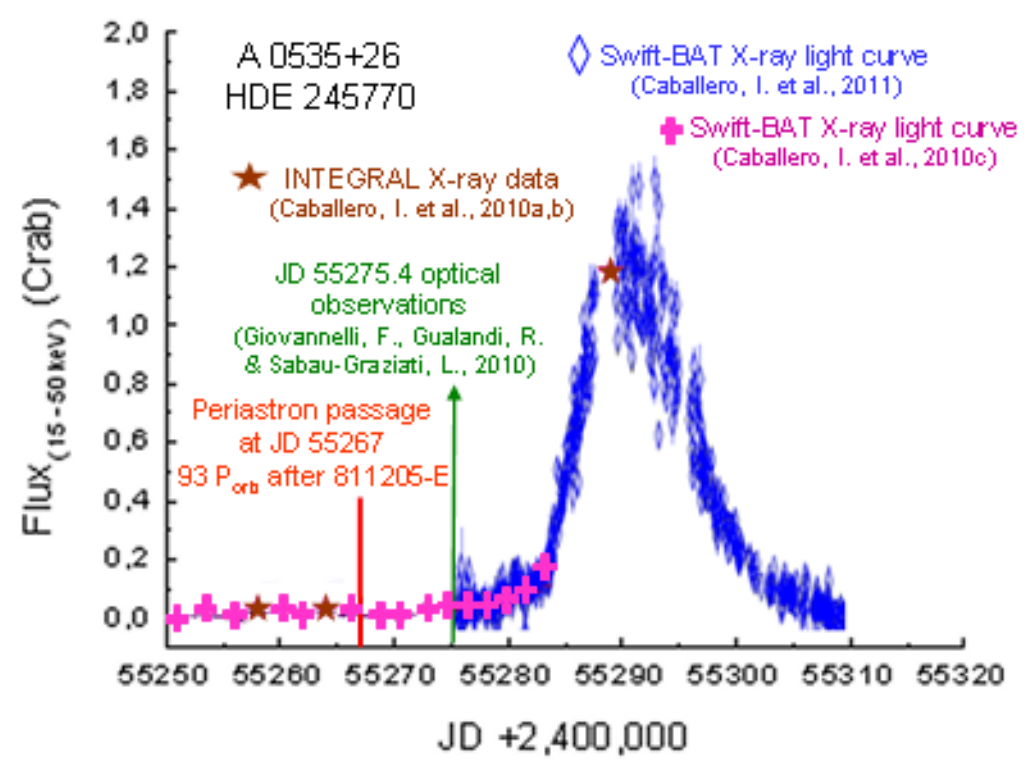

Figure 10: The predicted March-April 2010 X-ray outburst of A 0535+26 (Giovannelli, Gualandi \& SabauGraziati, 2010) after the 93th passage at the periastron after 811205-E (Caballero et al., 2010a,b,c,d; Caballero et al., 2011).

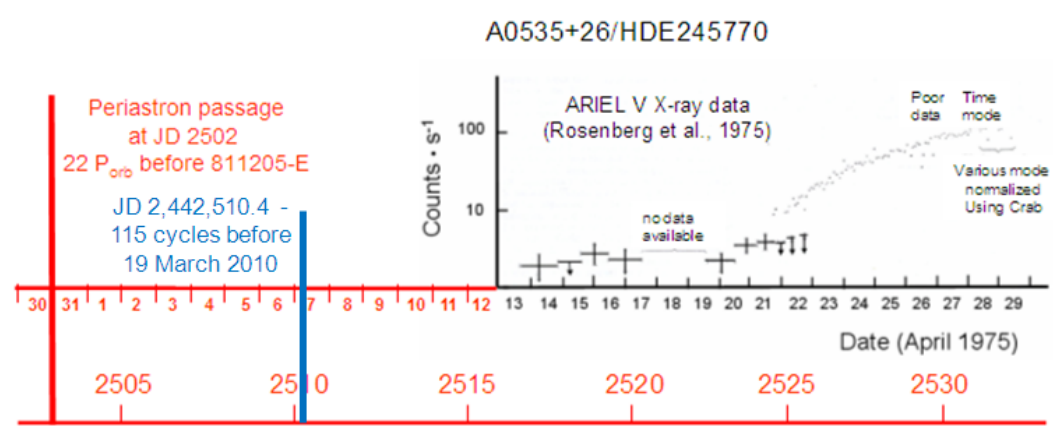

$\mathrm{JD}+2,440,000$

Figure 11: The Periastron passage at the 22nd cycle before 811205-E (JD 2502) (red line) precedes of $\sim 14$ days the X-ray outburst of A 0535+26 which starts approximatively on JD 2516 (after Rosenberg et al., 1975). The vertical blue line indicates the day April 7, 1975 (JD 2,442,510.4 ) just 115 cycles before the strong optical spectroscopic activity detected on March 19, 2010 (JD 2,455,275.4) (after GBK13).

are reported. Unfortunately in 1975, around the time of the discovery of A $0535+26$ no optical measurements are available for obvious reasons. The vertical red line indicates the time of the periastron passage following GBK13's ephemeris. This passage occurred at the 22nd cycle before the 811205-E. The vertical blue line indicates the day April 7, 1975 (JD 2,442,510.4 ) just 115 cycles before the strong optical spectroscopic activity detected on March 19, 2010 (JD 2,455,275.4), that preceded the strong X-ray outburst reported in Fig. 10.

The similarity between the first X-ray outburst and that of March-April 2010 is evident, and the separation of the two events is exactly 115 cycles. 


\section{General model of time lag between optical and X-ray outbursts in binary accreting sources}

In LMXBs (Low-Mass X-ray Binaries) the compact object can be either a neutron star or a black hole and the optical companion is a low mass star. The exchange of matter occurs via Roche lobe overflow, like shown in the left panel of Fig. 3. In HMXBs (High-Mass X-ray Binaries) the compact object can be either a neutron star or a black hole and the optical companion is a high mass star: giant or super-giant. The exchange of matter occurs mainly via stellar wind since usually the optical star does not fill its Roche lobe (Fig. 3, left panel). However, sometimes, the exchange of matter can occur in a mixed way because of the formation of an accretion disk around the compact object around the periatron passage (e.g. Giovannelli \& Ziółkowski, 1990) (Fig. 3, right panel).

McClintock, Narayan \& Rybicki (2004) found a very interesting relationship between the minimum X-ray luminosity in the range $0.5-10 \mathrm{keV}$ and the orbital periods of BH LMXBs and NS LMXBs. BH LMXBs are on average a factor of $\sim 100$ fainter than NS LMXBs with similar orbital periods (Fig. 12).

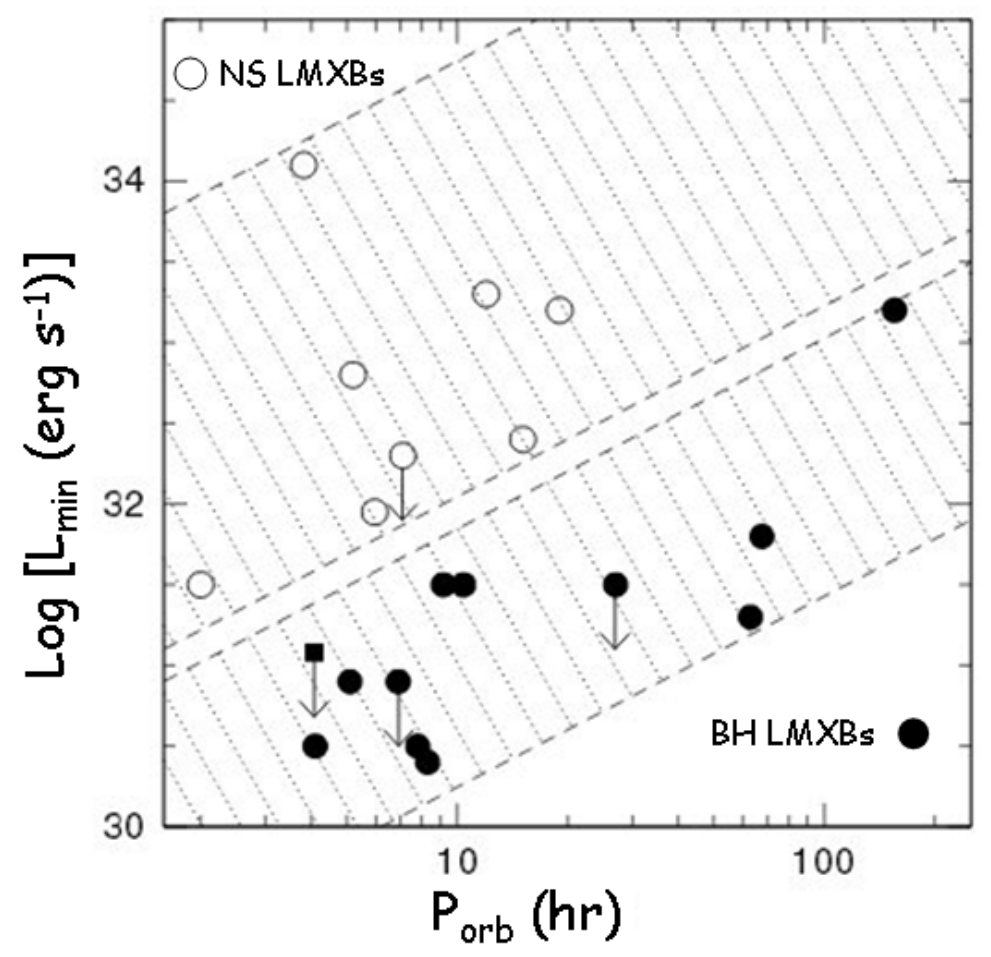

Figure 12: Minimum X-ray luminosity (0.5-10 keV) versus orbital period for BH LMXBs and NS LMXBs. The diagonal hatched areas delineate the regions occupied by the two classes of sources and indicate the dependence of luminosity on orbital period (adapted from McClintock, Narayan \& Rybicki, 2004).

As well known, X-ray/Be systems are formed by a compact star and an optical star. Obviously there is a mutual influence between the two stars. Low-energy (LE) processes influence highenergy (HE) processes and vice versa. Never confuse the effect with the cause. There is a general law in the Universe: Cause and Effect. The Cause generates an Effect and NOT vice versa! 
It is right to remind that the mechanism proposed by GBK13 for explaining the X-ray-optical delay in A $0535+26 /$ HDE 245770 is based on an enhanced mass flux propagation through the viscous accretion disk. This mechanism, known as UV-optical delay (the delay of the EUV flash with respect to the optical flash) was observed and modeled for cataclysmic variables (e.g. Smak, 1984; Lasota, 2001). Time delays have been detected also in several other X-ray transient binaries. This is the reason that urged Bisnovatyi-Kogan \& Giovannelli (2017, BKG17) to generalize the aforementioned model, developed for the particular case of A 0535+26/HDE 245770 (Flavia' star). This general model provides the formula (6.1) of the time delay between the optical and X-ray flashes appearance in transient cosmic accreting sources:

$$
\tau=6.9 \frac{\mathrm{m}^{2 / 3} \dot{\mathrm{m}}^{1 / 15}}{\alpha^{4 / 5}\left(\mathrm{~T}_{4}\right)^{28 / 15}}
$$

where:

$\mathrm{m}=\mathrm{M} / \mathrm{M}_{\odot} ; \quad \dot{\mathrm{m}}=\dot{\mathrm{M}} /\left(10^{-8} \mathrm{M}_{\odot} / \mathrm{yr}\right) ; \quad \mathrm{T}_{4}=\mathrm{T}_{0} / 10^{4} \mathrm{~K} ; \quad \alpha=$ viscosity, and

$\mathrm{T}_{0}=$ maximum temperature in optics.

By using this formula it is possible to obtain an excellent agreement between the experimental and theoretical delays found in:

- X-ray/Be system A0535+26/HDE245770: $\tau_{\text {exp }} \simeq 8$ days (GBK13); $\tau_{\text {th }} \simeq 8$ days;

- Cataclysmic variable SS Cygni; $\tau_{\exp }=0.9-1.4$ days (Wheatley, Mauche \& Mattei, 2003); $\tau_{\mathrm{th}} \simeq 1.35$ days;

- Low-mass X-ray binary Aql X-1/V1333 Aql: $\tau_{\exp } \sim 3$ days (Shahbaz et al., 1998); $\tau_{\text {th }} \simeq$ 3.2days

- Black hole X-ray transient GRO J1655-40: $\tau_{\exp } \sim 6$ days (Orosz et al., 1997); $\tau_{\text {th }} \simeq 6.5$ days.

In this general formula the $\alpha$-viscosity parameter plays an important role, and usually it is hard to be determined. However, if the other parameters are known, because experimentally determined, the formula (6.1) can be used for determining $\alpha$, taking into account the experimental delay measured in a certain source.

Over the last couple of decades we have witnessed the discovery of a multitude of highly ionized absorbers in high-resolution X-ray spectra from both BH and NS XRBs. The first detections were obtained thanks to ASCA on the BH binaries GROJ1655-40 and GRS 1915+105. Narrow absorption lines in the spectra of these systems identified as Fe XXV and Fe XXVI indicated the first of many discoveries of photo-ionized plasmas in LMXBs (Chandra, XMM-Newton and Suzaku). Black hole hot accretion flows occur in the regime of relatively low accretion rates and are operating in the nuclei of most of the galaxies in the universe. One of the most important progress in recent years in this field is about the wind or outflow. This progress is mainly attributed to the rapid development of numerical simulations of accretion flows, combined with observations on, e.g., Sgr A*, the SMBH in the Galactic center. The mass loss from a $\mathrm{BH}$ via wind is related to the mass accretion rate onto the $\mathrm{BH}$ as (Yuan, 2016): 


$$
\dot{\mathrm{M}}_{\text {wind }}(\mathrm{r})=\dot{\mathrm{M}}_{\mathrm{BH}} \times\left(\mathrm{r} / 20 \mathrm{r}_{\mathrm{g}}\right)^{\mathrm{s}} \quad \text { with } \quad \mathrm{s} \approx 1 \quad \text { and } \quad \mathrm{r}_{\mathrm{g}}=\frac{2 \mathrm{GM}_{\mathrm{BH}}}{\mathrm{c}^{2}}
$$

At this point it is useful to make a sort of summary about the number of XRBSs, including CVs. Liu, van Paradijs \& van den Heuvel (2006, 2007) and Ziółkowski (2013) report 315 galactic XRBs: 197 LMXBs (63\%) and 118 HMXBs (37\%), 72 of which are Be/X-ray systems; moreover there are 62 BH candidates. Coleiro \& Chaty (2013) report that in the Milky way there are $\geq 200$ HMXBs. Ritter \& Kolb (2003) catalogue, in the 7.20 (Dec. 2013) version, reports 1166 CVs. Buckley (2015) reports about the discoveries of 530 new CVs from MASTER-Network and 855 CVs from Catalina Real Time Survey (CRTS) (http://nesssi.cacr.caltech.edu/DataRelease/). Ferrario, de Martino \& Gänsicke (2015) report the number of MCVs as $\approx 250$, and $\sim 60$ of which IPs, and considering those systems for which the magnetic field intensity has not yet been determined, their number is of $\approx 600$. Table 1 shows the content of XRBSs, including CVs, in the Galaxy, and in the LMC and SMC (Ziółkowski, 2013; Ferrario, de Martino \& Gänsicke, 2015; Buckley, 2015). The mass are expressed in unit of SMC.

Table 1: Comparison of numbers of different classes of X-ray Binary Systems in the Milky Way and in the Magellanic Clouds (Ziółkowski, 2013; Ferrario, de Martino \& Gänsicke, 2015; Buckley, 2015).

\begin{tabular}{|l|c|c|c|}
\hline Name of the Class & Milky Way & LMC & SMC \\
\hline $\begin{array}{l}\text { Total mass of the galaxy } \\
\text { (in MSMC units) }\end{array}$ & 100 & 10 & 1 \\
\hline High Mass X-ray Binaries & 118 & 26 & 83 \\
in this Be/X-ray & 72 & 19 & 79 \\
\hline Low Mass X-ray Binaries & 197 & 2 & - \\
\hline Black Hole Candidates & 62 & 2 & - \\
\hline Cataclysmic Variables & $\approx 2000$ & - & - \\
in this MCVs & $\approx 250$ & - & - \\
IPs & $\sim 60$ & - & - \\
B not yet determined & $\approx 600$ & - & - \\
\hline
\end{tabular}

Grimm (2003) published: (i) a list of the 17 most luminous LMXBs contributing $\approx 90 \%$ to the integrated luminosity of LMXBs in the 2-10 keV band in the whole Galaxy, averaged over 19962000. The 12 most luminous sources (Cir X-1, GRS 1915+105, Sco X-1, Cyg X-2, GX 349+2, GX 17+2, GX 5-1, GX 340+0, GX 9+1, NGC 6624, Ser X-1, GX 13+1) contribute $\approx 80 \%$ of the integrated luminosity of the Galaxy; (ii) a list of the 10 most luminous HMXBs (Cyg X-3, Cen X-3, Cyg X-1, X 1657-415, V 4641 Sgr, GX 301-2, XTE J1855-024, X 1538-522, GS 1843+009, $\mathrm{X} 1908+075$ - that contribute $\approx 40 \%$ to the integrated luminosity of HMXBs in the $2-10 \mathrm{keV}$ band in the whole Galaxy, averaged over 1996-2000.

Raguzova \& Lipunov (1999) - using the "Scenario Machine" developed by Lipunov (1987) and Lipunov \& Postnov (1988) - obtained an evolutionary track that can lead to the formation of 
$\mathrm{Be} / \mathrm{BH}$ systems. This result has been confirmed fifteen years later by Casares et al. (2014) who discovered MWC 656, the first Be/BH binary.

Indeed, Raguzova \& Lipunov (1999) calculations show that binary black holes with Be stars must have $0.2<\mathrm{e}<0.8$. It is particularly difficult to detect such systems as most of their spectroscopic variations occur in a relatively small portion of the orbit, and could easily be missed if the systems are observed at widely separated epochs. This represents one more reason for asking continuous multifrequency observations of different classes of cosmic sources in order to understand their true behaviour.

The critical initial mass of the supernova star that collapses to a $\mathrm{BH}$ is accepted to be equal to $55<\mathrm{M}_{\mathrm{cr}}<75 \mathrm{M}_{\odot}$, and the fraction of the presupernova mass $\left(\mathrm{M}_{\star}\right)$ collapsing to the $\mathrm{BH}, \mathrm{k}_{\mathrm{BH}}=$ $\mathrm{M}_{\mathrm{BH}} / \mathrm{M}_{\star}=0.5$. The kick velocity $\mathrm{v}_{\mathrm{m}}=0-200 \mathrm{~km} \mathrm{~s}^{-1}$. The age of the system, according to their evolutionary scenario is $4 \times 10^{6} \mathrm{yr}$.

The expected number of Be/BH binaries - with orbital period $10 \mathrm{~d}<\mathrm{P}_{\text {orb }}<1000 \mathrm{~d}$, and eccentricity $0.2<\mathrm{e}<0.8-$ is $1 \mathrm{Be} / \mathrm{BH}$ for $20-30 \mathrm{Be} / \mathrm{NS}$.

Belczynski and Ziółkowski (2009) used binary population synthesis models to show that the expected ratio of Be/XRBs with neutron stars to black holes in the Galaxy is relatively high ( $30-50)$, and so broadly in line with observations. Thus we can expect $1 \mathrm{Be} / \mathrm{BH}$ for 30-50 Be/NS.

Therefore, we can expect $1 \mathrm{Be} / \mathrm{X}$-ray BH system for 20-50 Be/X-ray NS systems (Raguzova \& Lipunov, 1999; Belczynski \& Ziółkowski, 2009). We know 60 Be/X-ray NS systems (after INTEGRAL). Thus we expect 1-3 Be/X-ray BH systems. One of this systems has been detected: MWC 656 (Casares et al., 2014).

New simulations - using the StarTrack binary population synthesis models have been conducted to understand the formation channel of MWC 656 - constrain the population of $\mathrm{Be} / \mathrm{BH}$ systems and study the fate of MWC 656 as a possible NS-BH merger, and then possible gravitational wave emitter. In particular, it has been assumed that all donors beyond main sequence are allowed to survive the common envelope (CE) phase. Ten Gyr of evolution of the Galactic disk originates $\sim 8700 \mathrm{~B} / \mathrm{BH}$ systems, and 1/3 of them would be Be/BH systems: namely $\sim 2900$ ). However, only 13 of them had periods, eccentricities and masses similar to MWC 656 (Grudzinska et al., 2015).

There are so many black holes in the Universe that it is impossible to count them.

Stellar-mass black holes (SBHs) form from the most massive stars when their lives end in supernova explosions. The Milky Way galaxy contains some $10^{11}$ stars. Roughly one out of every thousand stars that form is massive enough to become a black hole. Therefore, our galaxy must harbor some $10^{8}$ stellar-mass black holes. Most of these are invisible to us, and only nineteen have been identified (Wiktorowicz, Belczynski \& Maccarone, 2014) with masses up to $\sim 15 \mathrm{M}_{\odot}$. Theoretically the mass of a SBH depends on the initial mass of the progenitor, how much mass is lost during the progenitor's evolution and on the supernova explosion mechanism (Belczynski et al., 2010; Fryer et al., 2012). Mass is lost through stellar winds, and the amount of mass lost strongly depends on the metallicity of the star. For a low metallicity star $(\sim 0.01$ of the solar metallicity) it is possible to leave a black hole of $\leq 100 \mathrm{M}_{\odot}$ (Belczynski et al., 2010). In the region of the Universe visible from Earth, there are perhaps $10^{11}$ galaxies. Each one has about $10^{8}$ stellar-mass black holes. And somewhere out there, a new stellar-mass black hole is born in a supernova every second. 
However, some attempts of evaluation of the number of SBHs in the Galaxy have been done. For instance, taking into account the $\gamma$-ray emissivity of the Galaxy $\left(1.3 \times 10^{43} \mathrm{~s}^{-1}\right.$ for $\mathrm{E}>100$ $\mathrm{MeV}$ ) measured by the SAS II satellite (Strong, Wolfendale \& Worral, 1976) and the processes of disk-fed accretion onto black holes, Giovannelli, Karakuła \& Tkaczyk $(1981,1982)$ - considering a spherical accretion flow with a constant Mach number, corresponding to the adiabatic power $\gamma=$ $5 / 3$ - found a possible upper limits to the number of black holes $\left(\mathrm{M} \sim 10 \mathrm{M}_{\odot}\right.$ and $\dot{\mathrm{M}} \approx 10^{-8} \mathrm{M}_{\odot}$ $\mathrm{yr}^{-1}$ ) of $10^{-5}-10^{-4}$ of the total star population of the Galaxy.

There is a class of intermediate-mass black holes (IMBHs), with masses $>100 \mathrm{M}_{\odot}$ up to $\approx 10^{5} \mathrm{M}_{\odot}$. It contains a dozen systems, as listed in Johnstone (2004). However, black holes with masses of several hundred to a few thousand solar masses remain elusive, as reported in a review by Casares \& Jonker (2014) where a deeply discussion about the mass measurements of SBHs and IMBHs is contained.

Supermassive black holes (SMBHs) are $10^{6}-10^{10}$ times more massive than our Sun and are found in the centers of galaxies (see the exhaustive review by Kormendy \& Ho, 2013). Most galaxies, and maybe all of them, harbor such a black hole. So in our region of the Universe, there are some $10^{11} \mathrm{SMBHs}$. The nearest one resides in the center of our Milky Way galaxy. The most distant one we know of resides in a quasar galaxy billions of lightyears away. SMBHs grow in size as they gorge on surrounding matter.

A list of BH candidates has been reported by Robert Johnston (2004) and provides the input for constructing the map of sky locations of BH candidates. Source list includes results reported in Kormendy \& Gebhardt (2001), Orosz (2002), Tremaine et al. (2002), and Ziolkowski (2003).

In the case of galactic compact sources, by using the softness and hardness ratios, coming for the measurements of the many X-ray satellites, it is possible to construct a diagram in which BHs in high state are separated by those in low state, and by other kind of objects, such as X-ray pulsars and other systems, as shown in Fig. 13 (Giovannelli, 2016, after Tanaka, 2001).

\section{Model of time lag between optical and X-ray outbursts in AGNs}

As already noted black hole accretion is a fundamental physical process in the universe. It is the standard model for the central engine of active galactic nuclei (AGNs), and also plays a central role in the study of black hole X-ray binaries, Gamma-ray bursts, and tidal disruption events. According to the temperature of the accretion flow, the accretion models can be divided into two classes, namely cold and hot. The standard thin disk model belongs to the cold disk, since the temperature of the gas is far below the virial value (Shakura \& Sunyaev, 1973) (see reviews by (Pringle, 1981; Frank, King \& Rayne, 2002; Bisnovatyi-Kogan, 2011; Abramowicz \& Fragile, 2013; Blaes, 2014). The disk is geometrically thin but optically thick and radiates multi-temperature black body spectrum. The radiative efficiency is high, $\sim 0.1$, independent of the accretion rate. The model has been successfully applied to luminous sources such as luminous AGNs and black hole X-ray binaries in the thermal state. The most recent review about the accretion onto black holes was published by Lasota (2016).

The tidal disruption of stars by massive BHs has been discussed since many years by Rees (1988), and e.g. Magorrian \& Tremaine (1999). Rees (1988) argued that stars in galactic nuclei can be captured or tidally disrupted by a central black hole. Some debris would be ejected at high 


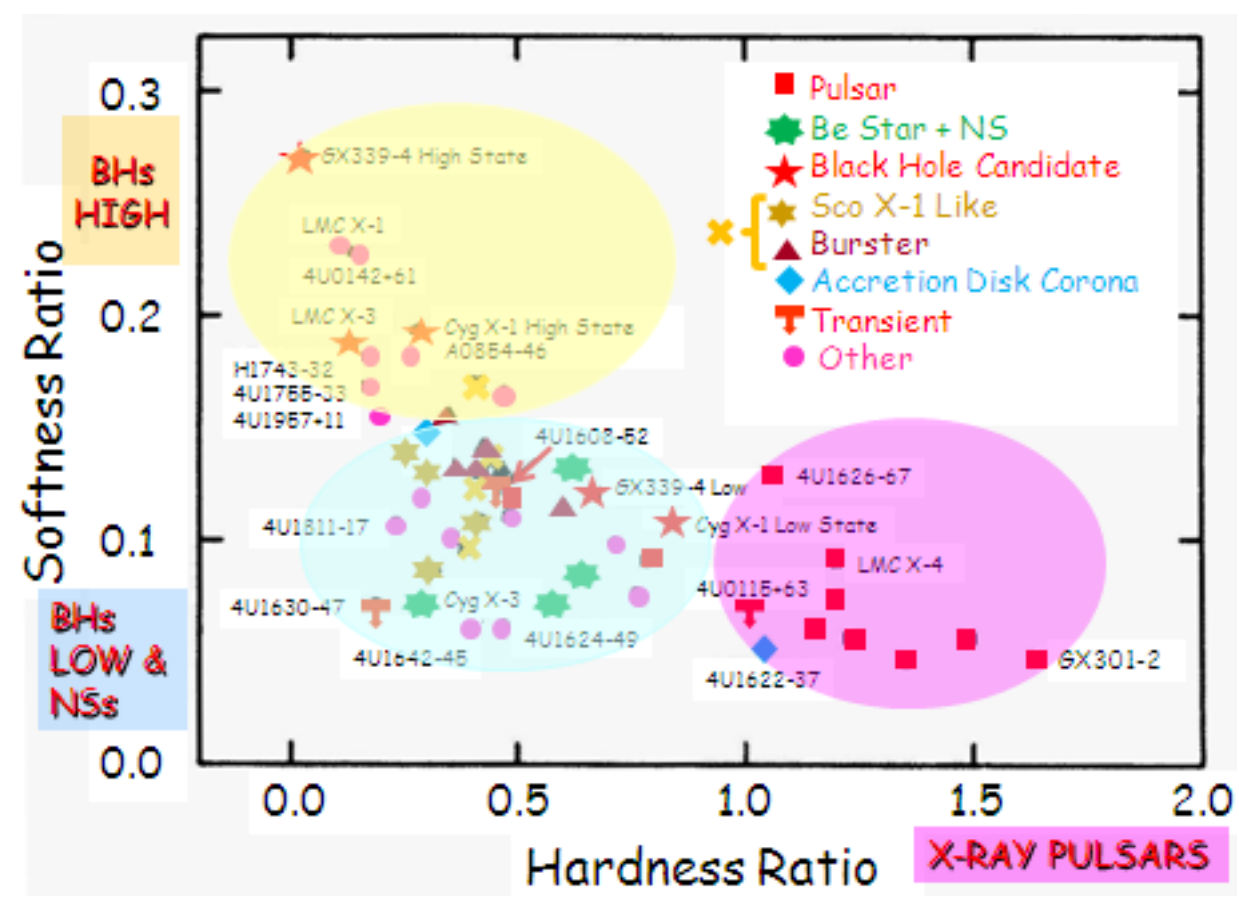

Figure 13: Softness ratio versus hardness ratio for galactic compact systems. Light yellow ellipse marks the zone where BHs in high state lie, light turquoise ellipse marks the zone of the BHs in low state and NSs, and light fuchsia ellipse marks the zone of the X-ray pulsars (Giovannelli, 2016, after Yasuo Tanaka, 2001).

speed, the remainder would be swallowed by the hole, causing a bright flare lasting at most a few years. Such phenomena are compatible with the presence of $10^{6}-10^{8} \mathrm{M}_{\odot}$ holes in the nuclei of many nearby galaxies. Stellar disruption may have interesting consequences in our own Galactic Center if $\mathrm{a} \approx 10^{6} \mathrm{M}_{\odot}$ hole lurks there.

In a recent paper, BKG17 developed models of time lags between optical and X-ray flashes for close-binary galactic sources with accretion disks and for an AGN with an SMBH that is embedded in a quasi-spherical bulge. The flashes in an AGN are considered in the model when a disruption of a star that is in the evolution phase of a giant enters the radius of strong tidal forces. The matter with a low angular momentum that is released by the star falls into the SMBH in the form of a quasi-spherical flow with a velocity that is close to the free-fall velocity. An X-ray flash occurs when the falling matter reaches the hot inner regions. The time lag observed in these sources is identified with the time of the matter falling from the tidal radius onto the central region. The values of the tidal radius that they calculated in this model were compared with the theoretical radii of a tidal disruption that depends on the masses of the SMBH and of the star, and on the radius of the star.

Knowing the SMBH masses from observations, and making a reasonable suggestion for the stellar mass that is on the order of one solar mass, they obtained that the radii of the disrupted star are between a few tens and a few hundreds of $R_{\odot}$ (see Table 2 ). These radii are characteristic of stars of moderate mass on the giant phase of evolution (e.g. Bisnovatyi-Kogan, 2011).

The time delay between the optical and X-ray flashes is experimentally determined. The radius 
at which the optical flash occurs is calculated for the motion with free-fall velocity $\mathrm{V}_{\mathrm{ff}}$ as:

$$
\mathrm{V}_{\mathrm{ff}}=(2 \mathrm{GM} / \mathrm{r})^{1 / 2} ; \mathrm{dr} / \mathrm{dt}=\mathrm{V}_{\mathrm{ff}} ; \tau_{\mathrm{ff}}=2 / 3\left[\mathrm{r}^{3 / 2} /(\mathrm{GM})^{1 / 2}\right]
$$

Taking $\tau_{\mathrm{ff}}=\tau_{\mathrm{obs}}$, BKG17 obtained a radius of the optical flash $\mathrm{r}_{\mathrm{opt}}$ as:

$$
\mathrm{r}_{\mathrm{opt}}=1.65 \times 10^{12} \tau_{\mathrm{obs}} \mathrm{m}^{1 / 3} \mathrm{~cm}
$$

where: $\tau_{\text {obs }}$ expressed in days, and SMBH mass: m expressed in $\left(\mathrm{M}_{\odot}\right)$.

Table 2: Properties of stars tidally disrupted by SMBH in AGNs (adapted from Bisnovatyi-Kogan \& Giovannelli, 2017). Here $m_{s}=\frac{\mathrm{M}_{\mathrm{s}}}{\mathrm{M}_{\odot}}$, where $\mathrm{M}_{\mathrm{S}}$ is the mass of the disrupted star.

\begin{tabular}{|l|c|c|c|}
\hline Source name & $\begin{array}{c}\tau_{\mathrm{obs}} \\
(\text { days })\end{array}$ & $\begin{array}{c}\mathrm{r}_{\mathrm{opt}}=\mathrm{r}_{t} \\
(\mathrm{~cm})\end{array}$ & $\begin{array}{c}\mathrm{R}_{s} \\
(\mathrm{~cm})\end{array}$ \\
\hline Mrk 509 & 15 & $5.2 \times 10^{15}$ & $114 \times m_{s}^{1 / 3} \mathrm{R}_{\odot}$ \\
\hline NGC 7469 & 4 & $8.95 \times 10^{14}$ & $47 \times m_{s}^{1 / 3} \mathrm{R}_{\odot}$ \\
\hline 3C 120 & $3.9-6.2 ?(10)$ & $3.3 \times 10^{15}$ & $100 \times m_{s}^{1 / 3} \mathrm{R}_{\odot}$ \\
\hline NGC 3516 & 100 & $1.1 \times 10^{16}$ & $409 \times m_{s}^{1 / 3} \mathrm{R}_{\odot}$ \\
\hline NGC 4051 & 2.4 & $2.5 \times 10^{14}$ & $36 \times m_{s}^{1 / 3} \mathrm{R}_{\odot}$ \\
\hline ASASSN-14li & 5 & $1.1 \times 10^{15}$ & $98 \times m_{s}^{1 / 3} \mathrm{R}_{\odot}$ \\
\hline
\end{tabular}

It is necessary to mention that another possibility to interpret the short time delays in AGNs is based on the irradiation model (e.g. Ulrich, Maraschi, \& Urry, 1997). This model could explain recent extensive observations of NGC 5548 in X-rays (SWIFT), UV, and optical light (HST) in the "reverberation mapping" campaign (Edelson et al., 2015; Fausnaugh et al., 2016).

However, with the formula (6.3) BKG17 justify the experimental time delay between optical and X-ray flashes observed in AGNs.

\section{Discussion and Conclusions}

We have discussed the genesis of the work that allowed us to develop models of time lags between optical and X-ray flashes for close-binary galactic sources with accretion disks and for an AGN with a SMBH that is embedded in a quasi-spherical bulge.

The time lag in disk-accreting galactic close-binary sources is based on a sudden increase in the accretion flow that starts at the disk periphery and is related to the optical maximum. The massive accretion layer propagates to the central compact source as a result of the turbulent viscosity. The $\mathrm{X}$-ray flash occurs when this massive layer reaches the inner hot regions of the accretion disk and falls into the central compact object. The matter in the accretion disk moves inside with a speed that is determined by the turbulent viscosity. We described this model quantitatively and derived an analytic formula that determines the value of the time lag. This formula gives results that agree well with observational values. The flashes in an AGN are considered in the model when a disruption of a star that is in the evolution phase of a giant enters the radius of strong tidal forces. The matter with a low angular momentum that is released by the star falls into the SMBH in the form of a quasi-spherical flow with a velocity that is close to the free-fall velocity. An X-ray flash occurs 
when the falling matter reaches the hot inner regions. The time lag observed in these sources is identified with the time of the matter falling from the tidal radius onto the central region. The values of the tidal radius that we calculated in this model were compared with the theoretical radii of a tidal disruption that depends on the masses of the SMBH and of the star, and on the radius of the star. Knowing the SMBH masses from observations, and making a reasonable suggestion for the stellar mass that is on the order of one solar mass, we obtained that the radii of the disrupted star are between a few tens and a few hundreds of $\mathrm{R}_{\odot}$. These radii are characteristic of stars of moderate mass on the giant phase of evolution (see, for instance, Bisnovatyi-Kogan, 2011).

The matter with larger angular momentum that appeared in the disruption of the star is expected to form an accretion disk through which the matter will move to the center as a result of turbulent viscosity, similarly to flashes in close galactic binaries. This motion is much slower than free-fall velocity and may last for many years. After such a flash in AGNs, we therefore expect a long-duration irregular variability in the whole electromagnetic spectrum.

The variability properties observed in many AGNs, where optical and UV emission lags the $\mathrm{X}$-ray light curve, may be explained by the model in which an X-ray flash in the center of AGN is followed by reradiation of the surrounding accretion disk.

Acknowledgments This research has made use of the NASA's Astrophysics Data System;

\section{References}

[1] Abramowicz, M.A., Fragile, P.C.: 2013, Living Rev. in Relativity 16, Issue 1, article id. 1, 88 pp.

[2] Baade, W., Zwicky, F.: 1934, Phys. Rev. 45, 138.

[3] Bartolini, C., Guarnieri, A., Piccioni, A., Giangrande, A., Giovannelli, F.: 1978, IAU Circ. No. 3167.

[4] Bartolini, C., Bianco, G., Guarnieri, A., Piccioni, A., Giovannelli, F.: 1983, Hvar Obs. Bull. 7(1), 159.

[5] Belczynski, K., Ziółkowski, J.: 2009, ApJ 707, 870.

[6] Belczynski, K., Dominik, M., Bulik, T., O’Shaughnessy, R., Fryer, C., Holz, D.E.: 2010, ApJL 715, L138.

[7] Bisnovatyi-Kogan, G.S.: 2011, Stellar Physics 2: Stellar Evolution and Stability, Berlin-Heidelberg: Springer-Verlag.

[8] Bisnovatyi-Kogan, G.S., Komberg, B.V.: 1974, Sov. Astron. 18, 217.

[9] Bisnovatyi-Kogan, G.S., Giovannelli, G.: 2017, A\&A 599, A55, 7 pp. (BKG17).

[10] Blaes, O.: 2014, SSRv 183, 21-41.

[11] Blumenthal, G.R., Tucker, W.H.: 1974, Ann. Rev. A\&A 12, 23-46.

[12] Buckley, D., 2015, talk at the Palermo Workshop on "The Golden Age of Cataclysmic Variables and Related Objects - III".

[13] Burbidge, E.Margaret, Burbidge, G.R., Fowler, W.A., Hoyle, F.: 1957, Rev. Mod. Phys. 29, 547-655.

[14] Burger, M., van Dessel, E.L., Giovannelli, F., Sabau-Graziati, L., Bartolini, C. et al.: 1996, in Multifrequency Behaviour of High Energy Cosmic Sources, F. Giovannelli \& L. Sabau-Graziati (eds.), Mem. SAIt. 67, 365. 
[15] Caballero, I., Lebrun, F., Rodriguez, J., Soldi, S., Mattana, F., et al.: 2010a, ATel. 2496.

[16] Caballero, I., Santangelo, A., Pottschmidt, K., Klochkov, D., Rodriguez, J., et al.: 2010b, ATel. 2541.

[17] Caballero, I., Pottschmidt, K., Barragán, L., Ferrigno, C., Klochkov, D., et al.: 2010c, Talk at CRSF Meeting, Tübingen 2010.

[18] Caballero, I., Kretschmar, P., Pottschmidt, K., Santangelo, A., Wilms, J., et al.: 2010d, AIPC 1248, 147.

[19] Caballero, I., Pottschmidt, K., Santangelo, A., Barragán, L., Klochkov, D., et al.: 2011, arXiv:1107.3417.

[20] Cameron, A.G.W.: 1958, Ann. Rev. Nucl. Part. Sci. 8, 299-326.

[21] Camero-Arranz, A., Finger, M.H., Wilson-Hodge, C.A., Jenke, P., Steele, I., et al.: 2012, ApJ 754, 20

[22] Casares, J., Jonker, P.G.: 2014, SSRv 183, 223-252.

[23] Casares, J., Negueruela, I., Ribó, M., Ribas, I., Paredes, J.M. et al.: 2014, Nature 505, Issue 7483, 378-381.

[24] Chartres, M., Li, F.: 1977, IAU Circ. No. 3154.

[25] Clay, J.: 1927, Proc. Nederlandsche Akad. v. Wet. 30, 1115.

[26] Coe, M. J., Carpenter, G.F., Engel, A.R., Quenby, J.J.: 1975, Nature 256, 630.

[27] Coe, M.J., Reig, P., McBride, V.A., Galache, J.L., Fabregat, J.: 2006, MNRAS 368, 447.

[28] Coleiro, A., Chaty, S.: 2013, ApJ 764, 185.

[29] Corbet, R.H.D.: 1984, A\&A 141, 91.

[30] Corbet, R.H.D.: 1986, MNRAS 220, 1047.

[31] Edelson, R., Gelbord, J.M., Horne, K. et al.: 2015, ApJ 806, 129.

[32] Fasano, A.: 2015, Comportamento spettrofotometrico della transiente X A0535+26/HDE245770, Bachelor's degree thesis, Sapienza University, Roma, Italy.

[33] Fausnaugh, M.M., Denney, K.D., Barth, A.J. et al.: 2016, ApJ 821, 56.

[34] Ferrario, L., de Martino, D., Gänsicke, B.T.: 2015, SSRv 191, 111-169.

[35] Finger, M.H., Wilson, R.B., Hagedon, K.S. 1994 IAU Circ. No. 5931.

[36] Finger, M.H., Wilson, R.B., Harmon, B.A.: 1996, ApJ 459, 288.

[37] Finger, M.H., Camero-Arranz, A., Kretschmar, P., Wilson, C., and Patel, S.: 2006, BAAS 38, 359.

[38] Frank, J., King, A.R., Raine, D.J.: 1985, Accretion power in astrophysics, Cambridge and New York, Cambridge University Press, 283 pp.

[39] Fryer, C.L., Belczynski, K., Wiktorowicz, G., Dominik, M., Kalogera, V., Holz, D.E.: 2012, ApJ 749, 91.

[40] Ghosh, P.: 1994, in The Evolution of X-Ray Binaries, S.S. Holt \& C.S. Day (Eds.), AIP Conf. Proc. $308,439$.

[41] Giacconi, R., Gursky, H., Paolini, F.R., Rossi, B.: 1962, Phys. Rev. Lett. 9, 439.

[42] Giacconi, R., Gursky, H., Kellog, E., Schreier, E., Tananbaum, H.: 1971, Astrophys. J. Lett. 167 , L67. 
[43] Giacconi, R., Branduardi, G., Briel, U., Epstein, A., Fabricant, D.: 1979, ApJ. 230, 540.

[44] Giangrande, A., Giovannelli, F., Bartolini, C., Guarnieri, A., Piccioni, A.: 1980, A\&A Suppl. Ser. 40, 289.

[45] Giovannelli, F.: 2000, in The Evolution of The Milky Way, F. Matteucci \& F. Giovannelli (Eds.), Kluwer Academic Publishers, pp. 619-620.

[46] Giovannelli, F.: 2005, The Impact of Multifrequency Observations in High Energy Astrophysics, Ph. D. Thesis, University of Barcelona, Spain.

[47] Giovannelli, F.: 2015, talk at the Frascati Workshop 2015 on Multifrequency Behaviour of High Energy Cosmic Sources - XI, 29th May.

[48] Giovannelli, F.: 2016, in Proc. of the 4th Ann. Conf. on High Energy Astrophysics in Southern Africa (HEASA 2016). Online at http://pos.sissa.it/cgi-bin/reader/conf.cgi?confid=275, id.31

[49] Giovannelli, F., Karakuła, S., Tkaczyk, W.: 1981, in Origin of Cosmic Rays, Setti, G., Spada, G. \& Wolfendale, A.W. (Eds.), IAU Symp. 94, p. 335.

[50] Giovannelli, F., Karakuła, S., Tkaczyk, W.: 1982, Acta Astron. 32, 121.

[51] Giovannelli, F., de Loore, C., Bartolini, C., Burger, M., Ferrari-Toniolo, M., et al. 1982 in Proc. of the Third European IUE Conference, ESA SP-176, 233.

[52] Giovannelli, F., Ferrari-Toniolo, M., Persi, P., Golynskaya, I.M., Kurt, V.G., et al.: 1985 in Multifrequency Behaviour of Galactic Accreting Sources, F. Giovannelli (ed.), Edizioni Scientifiche SIDEREA, Roma, p. 284.

[53] Giovannelli, F., Ziółkowski, J.: 1990, AcA 40, 95-103.

[54] Giovannelli, F., Sabau-Graziati, L.: 1992, SSRv 59, 1-81.

[55] Giovannelli, F., Sabau-Graziati, L.: 2001, Ap\&SS, 276, 67-80.

[56] Giovannelli, F., Bernabei, S., Rossi, C., Sabau-Graziati, L.: 2007, A\&A, 475, 651.

[57] Giovannelli, F., Gualandi, R., Sabau-Graziati, L.: 2010, ATel. 2497.

[58] Giovannelli, F., Sabau-Graziati, L.: 2011, Acta Polytechnica Vol. 51, No. 2., p. 21.

[59] Giovannelli, F., Bisnovatyi-Kogan, G.S., Klepnev, A.S.: 2013, A\&A 560, id.A1, 11 pp (GBK13).

[60] Giovannelli, F., Bisnovatyi-Kogan, G.S., Bruni, I., Corfini, G., Martinelli, F., Rossi, C.: 2015a, AcA 65, 107-116.

[61] Giovannelli, F., Rossi, C., Bisnovatyi-Kogan, G., Bruni, I., Fasano, A., Salas Procas, J.: 2015b, Proc. of the XI Multifrequency Behaviour of High Energy Cosmic Sources Workshop (MULTIF15). Online at http://pos.sissa.it/cgi-bin/reader/conf.cgi?confid=246, id.39

[62] Giovannelli, F., Sabau-Graziati, L.: 2016, in Frontier Research in Astrophysics II, Online at https://pos.sissa.it/cgi-bin/reader/conf.cgi?confid=269, id. 1.

[63] Grimm, H.-J.: 2003, PhD Thesis, Ludwig-Maximilians-Universitat, München, Germany.

[64] Grudzinska, M., Belczynski, K., Casares, J., de Mink, S.E., Ziolkowski, J.: 2015, MNRAS, 452, 2773-2787.

[65] Guarnieri, A., Bartolini, C., Piccioni, A., Giovannelli, F.: 1985b, in Multifrequency Behaviour of Galactic Accreting Sources, F. Giovannelli (ed.), Frascati: CNR, Istituto di Astrofisica, Edizioni Scientifiche SIDEREA, Roma, p. 310. 
[66] Hutchings, J.B.: 1984, PASP 96, 312.

[67] Hayakawa, S.: 1952, Prog. Theor. Phys. 8, 571.

[68] Hess, V.F.: 1912, Physik Zh. 13, 1084.

[69] van den Heuvel, E.P.J., Rappaport, S.: 1987, in Physics of Be Stars, A. Slettebak \& T.P. Snow (eds.), Cambridge and New York, Cambridge University Press, Proc. of the IAU Coll. N. 92, p. 291.

[70] Janot-Pacheco, E., Motch, C., Mouchet, M.: 1987, A\&A 177, 91.

[71] Johnstone, Wm. R.: 2004, http://www.johnstonsarchive.net/relativity/bhctable.html

[72] Joss, P.C., Rappaport, S.A.: 1984, Ann. Rev. Astron. Astrophys. 22, 537.

[73] Kaluzienski, L.J., Holt, S.S., Boldt, E.A. Serlemitsos, P.J.: 1975, Nature 256, 633.

[74] van Kerkwijk, M.H., van Paradijs, J., Zuiderwijk, E.J.: 1995, A\&A 303, 497.

[75] Kormendy, J., Gebhardt, K.: 2001, arXiv:astro-ph/0105230v1.

[76] Kormendy, J., Ho, L.C.: 2013, Ann. Rev. A\&A 51, 511-653.

[77] Lasota, J.-P.: 2001, New Astron. Rev. 45, 449.

[78] Lasota, J.-P.: 2016, in Astrophysics of Black Holes, Cosimo Bambi (Ed.), Astrophysics and Space Science Library, Volume 440. ISBN 978-3-662-52857-0. Springer-Verlag Berlin Heidelberg, p. 1.

[79] Lipunov, V.M.: 1987, Ap\&SS 132, no. 1, 1-51.

[80] Lipunov, V.M., Postnov, K.A.: 1988, Ap\&SS 145, no. 1, 1-45.

[81] Liu, Q.Z., van Paradijs, J., van den Heuvel, E.P.J.: 2006, A\&A 455, 1165.

[82] Liu, Q.Z., van Paradijs, J., van den Heuvel, E.P.J.: 2007, A\&A 469, 807.

[83] de Loore, C., Giovannelli, F., van Dessel, E.L., Bartolini, C., Burger, M., et al.: 1984 A\&A 141, 279.

[84] Magorrian, J., Tremaine, S.: 1999, MNRAS 309, 447-460.

[85] McClintock, J.E., Narayan, R., Rybicki, G.B.: 2004, ApJ 615, 402-415.

[86] Margon, B., Nelson, J., Chanan, G., Bowyer, S., Thorstensen, J.R.: 1977, ApJ 216, 811.

[87] de Martino, D., Vittone, A., Giovannelli, F., Ciatti, F., Margoni, R., et al.: 1985, in Multifrequency Behaviour of Galactic Accreting Sources, Proceedings of the 1984 Frascati Workshop, Franco Giovannelli (ed.). Frascati: CNR, Istituto di Astrofisica, Ed. Scientifiche SIDEREA, Roma, p. 326

[88] de Martino, D., Waters, L.B.F.M., Giovannelli, F., Persi, P.: 1989, in The 23rd ESLAB Symposium on Two Topics in X Ray Astronomy, Volume 1 X-Ray Binaries, ESA SP 296, 519-520.

[89] Motch, C., Stella, L., Janot-Pacheco, E., Mouchet, M.: 1991, ApJ 369, 490.

[90] Nagase, F.: 1989, PASJ 41, no. 1, 1-79.

[91] Nagase, F., Hayakawa, S., Kunieda, H., Makino, F., Masai, K., et al.: 1982, ApJ 263, 814.

[92] Orosz, J.A.: 2002, arXiv:astro-ph/0209041v1.

[93] Orosz, J.A., Remillard, R.A., Bailyn, C.D., McClintock, J.E.: 1997, ApJL 478, L83.

[94] Peterson, L., Winckler, J.R.: 1958, Phys. Rev. Lett. 1, Issue 6, 205-206. 
[95] Piccioni, A., Bartolini, C., Bernabei, S., Guarnieri, A., Tarozzi, F., Valentini, G.: 1999, in Frontier Objects in Astrophysics and Particle Physics, F. Giovannelli \& G. Mannocchi (Eds.), SIF, Bologna, Italy, 65, 195.

[96] Priedhorsky, W.C., Terrell, J.: 1983, Nature 303, 681.

[97] Pringle, J.E.: 1981, Ann. Rev. A\&A 19, 137-162.

[98] Raguzova, N.V., Lipunov, V.M.: 1999, A\&A 349, 505.

[99] Rappaport, S., Joss, P.C.: 1981, in X-Ray Astronomy with the Einstein Satellite, R. Giacconi (ed.), D. Reidel Publ. Co., Dordrecht, Holland, p. 123.

[100] Rees, M.J.: 1988, Nature, 333, 523-528.

[101] Ricketts, M.J., Turner, M.J.L., Page, C.G., Pounds, K.A.: 1975, 256, 631-633.

[102] Ritter, H., Kolb, U.: 2003, A\&A 404, 301.

[103] Rosenberg, F.D., Eyles, C.J., Skinner, G.K., Willmore, A.P.: 1975, Nature 256, 628.

[104] Shahbaz, T., Bandyopadhyay, R.M., Charles, P.A., Wagner, R.M., Muhli, P., et al.: 1998, MNRAS $300,1035$.

[105] Shakura, N.I., Sunyaev, R.A.: 1973, A\&A 24, 337.

[106] Skinner, G.K.,, Bedford, D.K., Elsner, R.F., Leahy, D., Weisskopf, M.C., Grindlay, J.: 1982, Nature, 297, 568-570.

[107] Smak, J.: 1984, PASP 96, 5.

[108] Strong, A.W., Wolfendale, A.W., Worral, D.M.: 1976, MNRAS 175, 23.

[109] Tanaka, Y.: 2001, in The Century of Space Science, J.A. Bleeker, J. Geiss \& M. Huber (Eds.), Kluwer Academic Publishers, pp. 839-856.

[110] Thorsett, S.E., Arzoumanian, Z., McKinnon, M.M., Taylor, J.H.: 1993, ApJ 405, L29.

[111] Tremaine, S., Gebhardt, K., Bender, R., Bower, G., Dressler, A. et al.: 2002, ApJ 574, 740-753.

[112] Ulrich, M.-H., Maraschi, L., Urry, C.M.: 1997, ARA\&A, 35, 445.

[113] Vaiana, G.S., Cassinelli, J.P., Fabbiano, G., Giacconi, R., Golub, R.: 1981, ApJ. 245, 163.

[114] Villard, P.: 1900, Compt. Rend. Acad. Sci. Paris 130, 1010.

[115] Wheatley, P.J., Mauche, C.W., Mattei, J.A.: 2003, MNRAS 345, 49.

[116] Wiktorowicz,G., Belczynski, K, Maccarone, T.J.: 2014, in Binary Systems, their Evolution and Environments, arXiv:1312.5924v2.

[117] Wilson, C.T.R.: 1900, Proc. Cambridge Phil. Soc. 11, 32.

[118] Wood, K.S., Meekins, J.F., Yentis, D.J., Smathers, H.W., Menutt, D.P.: 1984, Astrophys. J. Suppl. Ser. 56, 507.

[119] Yan, J., Li, H., Liu, Q.: 2012, ApJ 744, 1.

[120] Yuan, F.: 2016, in Astrophysics of Black Holes, C. Bambi (Ed.), ApSSLibrary 440, 152-168.

[121] Zel'dovich, Ya.B., Guseinov, O.Kh.: 1965, Sov. Phys. Doklady 10, 524.

[122] Ziółkowski, J.: 2003, arXiv:astro-ph/0307307v1. 
[123] Ziółkowski, J.: 2013, Acta Polytechnica Vol 53, Suppl., 665.

[124] Zwicky, F.: 1939, Phys. Rev. 55, 726.

\section{DISCUSSION}

DMITRY BISIKALO: What is the physical reason of mass-transfer changing in your "delay model"?

FRANCO GIOVANNELLI: The physical reason for X-ray/Be is connected with eccentric orbit of NS or BH in the binary system, where the accretion rate is increasing in the vicinity of the periastron of the orbit. For CV the increase of the accretion rate is, probably, connected with the development of instability in the outer parts of the accretion disk, leading to the turbulent state with high viscosity.

VICTOR DOROSHENKO: You detected HeI line in 1999 in A0535+26, but there was no X-ray outburst, so there seems to be persistent disc around the NS, which is consistent with our XMM results in quiescence. What do you think about it?

FRANCO GIOVANNELLI: Yes, it is possible. The INTEGRAL monitor also detected some activity in quiescence.

VICTOR DOROSHENKO: Did I understand correctly that there is no need for field amplification for magnetars?

FRANCO GIOVANNELLI: In our model the magnetic field is not important in the accretion disk and accretion flow. It determines only the inner boundary of the accretion disk around the NS. 